\title{
EarthShine: Observing our world as an exoplanet from the surface of the Moon
}

\author{
Patricia T. Boyd $\odot,{ }^{\text {a, } *}$ Emily L. Wilson $\odot,{ }^{b}$ Alan P. Smale $\odot,{ }^{a}$ \\ Pete Supsinskas, ${ }^{\text {c }}$ Timothy A. Livengood $\odot,{ }^{a, d}$ Tilak Hewagama, ${ }^{e}$ \\ Geronimo L. Villanueva $\odot$, ${ }^{f}$ Alexander Marshak, ${ }^{b}$ Nickolay A. Krotkov $\odot, b$ \\ Petr Pokorny $\odot,{ }^{\text {a,g }}$ Jay Bixler, ${ }^{\mathrm{c}}$ Jonathan D. Noland, ${ }^{\mathrm{c}}$ Guru Ramu, ${ }^{\text {a,h }}$ \\ Paul Cleveland, ${ }^{a, i}$ John Ganino, ${ }^{c}$ Murzy Jhabvala, ${ }^{e}$ \\ Elisa Quintana, ${ }^{\text {a }}$ Emily Gilbert $\odot,{ }^{\text {a,j }}$ Knicole Colón, ${ }^{\text {a }}$ Giada N. Arney, \\ Shawn D. Domagal-Goldman $\odot,{ }^{\mathrm{f}}$ Avi Mandell $\odot,{ }^{\mathrm{f}}$ Tom Barclay, ${ }^{\mathrm{a}, \mathrm{k}}$ \\ Marc Kuchner $\odot,{ }^{a}$ and Lesley Ott ${ }^{b}$ \\ ${ }^{a}$ NASA Goddard Space Flight Center, Astrophysics Science Division, Greenbelt, \\ Maryland, United States \\ ${ }^{\mathrm{b}}$ NASA Goddard Space Flight Center, Earth Sciences Division, Greenbelt, \\ Maryland, United States \\ ${ }^{c}$ Lawrence Livermore National Laboratory, Livermore, California, United States \\ ${ }^{\mathrm{d}}$ University of Maryland, Department of Astronomy, College Park, Maryland, United States \\ ${ }^{e}$ NASA Goddard Space Flight Center, Instrument Systems and Technology Division, Greenbelt, \\ Maryland, United States \\ ${ }^{\mathrm{f}}$ NASA Goddard Space Flight Center, Planetary Science Division, Greenbelt, \\ Maryland, United States \\ ${ }^{\mathrm{g}}$ The Catholic University of America, Department of Physics, Washington, DC, \\ United States \\ ${ }^{\mathrm{h}}$ Mach 33 Engineering, Laurel, Maryland, United States \\ ${ }^{i}$ Energy Solutions International, Laytonsville, Maryland, United States \\ ${ }^{j}$ University of Chicago, Department of Astronomy and Astrophysics, Chicago, Illinois, \\ United States \\ ${ }^{k}$ University of Maryland, Baltimore County, Center for Space Sciences and Technology, \\ Baltimore, Maryland, United States
}

\begin{abstract}
NASA's return to the Moon coincides with explosive growth in exoplanet discovery. Missions are being formulated to search for habitable planets orbiting other stars, making this the ideal time to deploy an instrument suite to the lunar surface to help us recognize a habitable exoplanet when we see it. We present EarthShine, a technically mature, three-instrument suite to observe the whole Earth from the Moon as an exoplanet proxy. EarthShine data will validate and improve models critical for designing missions to image and characterize exoplanets, thus informing observing strategies for flagship missions to directly image exoplanets. EarthShine will answer interconnected questions in Earth and lunar science, exoplanets, and astrobiology, related to the credo "follow the water." EarthShine can take advantage of current NASA programs to conduct science from the Moon with low-cost, mature space hardware to reduce risk and assure success. Like the 1968 Apollo Earthrise image of our home planet, lonely in the black sky, the appeal of EarthShine to a multidisciplinary array of researchers in Earth Science, Planetary Science, and astrophysics will maximize both its scientific impact and its impact on the general public. () The Authors. Published by SPIE under a Creative Commons Attribution 4.0 International License. Distribution or reproduction of this work in whole or in part requires full attribution of the original publication, including its DOI. [DOI: 10.1117/1.JATIS.8.1.014003]
\end{abstract}

Keywords: EarthShine; Earth; Moon; lunar; exoplanet; glint; methane; carbon dioxide; water vapor; infrared; heterodyne; optical camera.

Paper 21055 received May 10, 2021; accepted for publication Dec. 20, 2021; published online Jan. 24, 2022.

*Address all correspondence to Patricia T. Boyd, padi.boyd@nasa.gov 


\section{Introduction}

Over the past 25 years, the pace of discovery of exoplanets within our galaxy—planets orbiting stars other than our Sun —has been astonishing. Thousands of planets confirmed around a wide variety of stars, and the inference of billions more, newly invigorates humanity's ancient quest to search for and identify unambiguous signs of life elsewhere in the Universe, to know whether we are alone. NASA and other agencies envision accomplishing this goal with enormous telescopes in space equipped with direct imaging technology to block out light from the star, billions of times brighter than any orbiting planet it may illuminate, to then study spectral features in the remaining light reflected or emitted from the planet.

Planets and their host stars are variable in space and time, complicating the interpretation of their spectra. Will we recognize a habitable exoplanet when we see it? The Earth is the beststudied habitable planet to inform the observations to be obtained by future exoplanet missions. NASA's return to the Moon presents a unique opportunity to refine these plans. The lunar surface sees the whole disc of the Earth through its range of illumination phases, differing only from an exoplanet observation because of the imaging resolution that is possible at comparatively close range. From the Moon, Earth can be seen in reflected light, in thermal self-emission, and as it passes in front of bright stars or the Sun. Comparing observations from the lunar surface to simultaneous measurements from NASA's Heliophysics and Earth Science satellites, we can distinguish features of habitation in the "exoEarth" spectral signature from environmental drivers unrelated to life.

To guide the interpretation of future exoplanet data, we present EarthShine, a low-cost, technologically mature instrument suite that views Earth as an exoplanet proxy from the Moon, to validate and improve models that are critically important for designing future missions to image and characterize exoplanets. The suite designed to be carried to the lunar surface by a Commercial Lunar Payload Service (CLPS) provider (Fig. 1) consists of a wide-field optical imaging spectrometer [Camera for Hyperspectral Earth Imaging from the Lunar Surface (CHEILS)] to acquire spectroscopic data cubes of Earth's disc, and a wide-field laser heterodyne radiometer (WF-LHR) for high-resolution lunar water vapor measurements to characterize any potential lunar exosphere contributions, and a mid- to long-wave infrared multispectral imager [Earth polychromatic camera-infrared (EPyC-IR)] to characterize Earth and correlate with the visible band in the exoEarth context. The spectral range of the combined instrument suite is 0.4 to $12.5 \mu \mathrm{m}$.

Results will form the basis to inform exoplanet observing strategies for future missions such as ASTRO2020 Decadal mission studies that focus on searches for habitable environments by directly imaging and characterizing planets orbiting other stars. The search for life on exoplanets is a driving goal for NASA and relies on detecting a distant, yet faint, world, and observing spectral signatures of gases such as methane and water vapor from the planet as a whole - gases the combined presence of which might indicate a habitable environment and perhaps even microbial activity. The lunar surface offers an accessible platform from which to refine these plans by taking advantage of the Earth as a habitable exoplanet laboratory where we can hone our

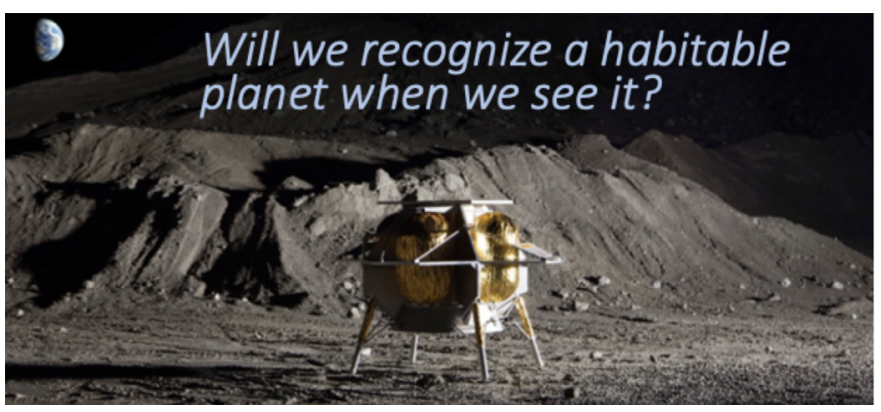

Fig. 1 EarthShine will see Earth from dusk to dawn on the Moon's nearside. Earth has not yet been systematically observed at high phase angle, the position in which half of all exoplanet observations will take place. EarthShine measurements will also apply to climate models and lunar water history. 
(a)

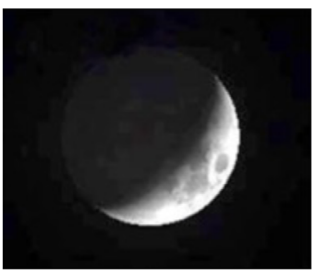

(b)

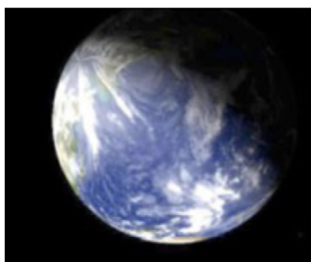

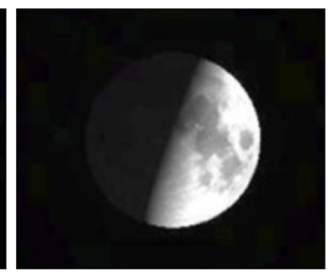

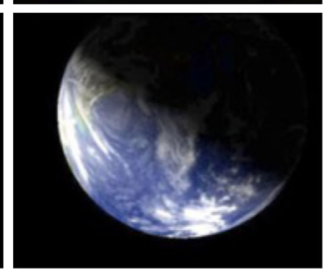

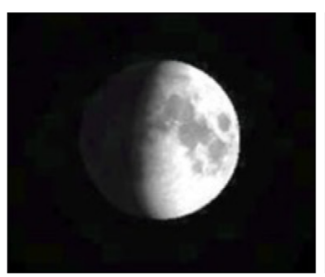

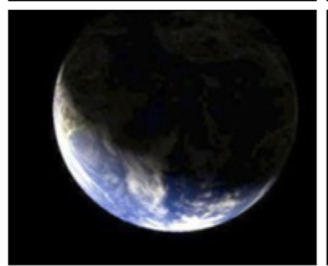

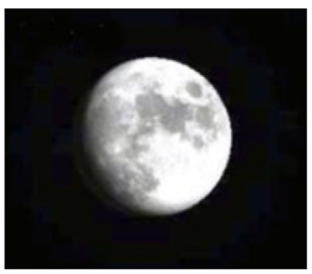

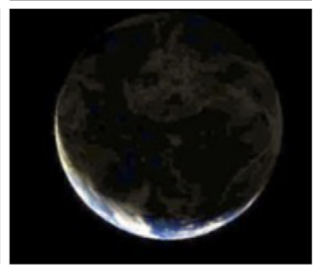

Fig. 2 The Moon's nearside enables observations of Earth phase and rotation. Complementary phases of Earth (b) as seen from Moon (a) allow EarthShine to access Earth's full rotation through unique illumination phases not achievable from GEO, LEO, or Earth-Sun Lagrange point (L1) orbits, in a single lunar day. Images: Stellarium, B. King, Sky \& Telescope, 2018 October. ${ }^{1}$

approaches to detect Earth-like life signatures on other worlds, including disentangling variability due to non-living components (projected ocean, ice and continent cover, cloud, and volcanic dynamics) from those changes due to the dynamic biosphere.

As the best studied and most extensively characterized planetary body, Earth is the critical anchor to validate and constrain models we will use to understand Earth-like exoplanets. Future spectra from these planets will come from a single-pixel combining numerous spatially and temporally varying components. They will present a variety of partially illuminated reflectance phases as the planet orbits the star, complicating data interpretation. EarthShine will continuously observe Earth's disc from the Moon, with two wide-field cameras collecting images and spectra from blue through thermal IR at phase angles inaccessible to earlier missions but essential for comparing with remote observations of exoplanets (Fig. 2). The laser heterodyne radiometer will search for water vapor on the lunar surface and for the purposes of simulations in this paper, we have assumed a site within the lunar swirl Reiner Gamma. Lunar swirls are features on the Moon's surface ranging from tens of meters to tens of kilometers with higher optical albedo and localized magnetic field anomalies. Addition of this long-pathlength surface observation will both constrain lunar exospheric water contributions to observations of Earth and contribute to understanding the history of water in our solar system and others-goals directly relevant to NASA's Artemis program.

As seen from the Moon, Earth occults bright stars and other Solar System planets several times per month. EarthShine can obtain data as these transitions "through" (behind) the Earth's atmosphere. Data from Earth occultations of bright sources can characterize the response of Earth's atmosphere, resulting in a database of star-planet observations to compare with exoplanet transmission spectroscopy.

Earth observations from the Moon will also complement low Earth orbit (LEO), geosynchronous equatorial orbit (GEO), and Earth-Sun L1 Lagrange point satellite measurements by providing comprehensive (in both time and space, compared to LEO) whole-globe monitoring, including polar regions not covered by GEO missions. Although L1 measurements do not capture a sufficient range in illumination and emission angles for the interpretation of exoplanet surface reflectance and cloud/aerosol phase function, a wider range of phase angles is available from the lunar surface observations. This enables EarthShine to retrieve aerosol and cloud scattering phase functions, monitor vegetation characteristics and plant physiology, better estimate surface albedo, and perform simultaneous imaging of the day and night faces of Earth, depending on lunar phase. Earth observations from a lunar vantage point will provide invaluable complementary datasets for Earth Science that can be compared with LEO, GEO, and L1 measurements. The coarser but broader measurements of carbon dioxide, methane, and water vapor would provide the full context needed to support regionally targeted data from LEO satellites. 
Finally, data collected during different Earth day/night times can refine our understanding of the Earth's biosphere and technosphere.

\section{Benefits of EarthShine}

\subsection{Observing the Earth's Atmosphere to Inform Exoplanet Studies}

Direct imaging spectroscopy of Earth-sized exoplanets, a powerful tool with the ability to transform our view of our place in the cosmos, is at the heart of NASA studies for missions at the flagship and probe levels. These missions can fulfill their potential only if their optical and spectroscopic designs - and eventual observations - are grounded in comprehensive, analogous data sets. Specifically, we need spectral observations of Earth as a whole disk, observed over the course of several of the planet's rotations and taken at different phase angles. Although prior efforts have obtained parts of this data set, ${ }^{2-12}$ the necessary combination of observations has not been collected. EarthShine will fill this gap and allow us to better assess the ability of future telescopes to determine specific planetary properties, including rotation rate, presence/ abundance of oceans and continents, degree of cloud cover, and presence of vegetation. The superior assessment EarthShine enables can be incorporated into technology development investments and architecture trades of the next flagship missions.

The combined 0.4 to $12.5 \mu \mathrm{m}$ range of EarthShine's instrumentation spans the wavelength range under consideration for remote detection and characterization of terrestrial exoplanets. The combined measurements will enable the testing of all planned methodologies and the direct comparison of their sensitivity to geological, atmospheric, and biogenic features. Astrobiology combines elements of all these drivers and how they impact habitability and life. Observations of Earth have already informed exoplanet models and future exoplanet observatories. ${ }^{8,10,13-15}$ Exoplanets, meanwhile, allow us to study types of planets not found in our solar system and to derive planetary statistics and processes on a comprehensive scale.

EarthShine's wavelength range also enables assessing a number of key biosignatures and habitability indicators in Earth's spectrum. ${ }^{16,17}$ Table 1 shows important gaseous species and surface reflectance features accessible to EarthShine, some of which can only be measured from space. EarthShine will be able to measure several habitability indicators: water $\left(\mathrm{H}_{2} \mathrm{O}\right)$, carbon dioxide $\left(\mathrm{CO}_{2}\right)$, and glints from ocean and cloud ice crystals (Fig. 3); ${ }^{18}$ as well as biosignatures (oxygen $\left(\mathrm{O}_{2}\right)$, ozone $\left(\mathrm{O}_{3}\right)$, methane $\left(\mathrm{CH}_{4}\right)$, and the vegetation red edge.

- Water $\left(\mathrm{H}_{2} \mathrm{O}\right)$ is central to terrestrial habitability. The concept of the thermally habitable zone assumes stable liquid phase water at the surface for a remotely detectable biosphere. EarthShine will measure atmospheric water at NIR and thermal wavelengths.

- Carbon dioxide $\left(\mathrm{CO}_{2}\right)$ influences plausible temperatures and chemistry in climate models of exoplanets. The chemical balance between oxidized carbon $\left(\mathrm{CO}_{2}\right)$ and organic carbon $\left(\mathrm{CH}_{4}\right)$ can signal or rule out the presence of significant abiotic methane in an anoxic environment. EarthShine will measure $\mathrm{CO}_{2}$ concentrations at NIR and thermal wavelengths.

- Oxygen $\left(\mathrm{O}_{2}, \mathrm{O}_{3}\right)$ is a trace gas in chemical equilibrium environments, such as the atmosphere of Mars. Common molecular oxygen indicates ongoing biogenic production on "modern" Earth, meaning the most recent $\sim 1$ to 2 billion years.

- Methane $\left(\mathrm{CH}_{4}\right)$ is predominantly biogenic on modern Earth. Distinguishing biogenic and abiotic (geological) methane sources is important to detecting habitability.

- Vegetation red edge (VRE) is a reflectance signature of green vegetation chlorophyll, a sharp difference between low albedo at wavelengths shortward of $0.7 \mu \mathrm{m}$ and high albedo at wavelengths longward of $0.7 \mu \mathrm{m}$.

- Scattering phase function. EarthShine complements EPIC/DSCOVR measurements ${ }^{11}$ from the Earth-Sun Lagrange point (L1) and EPOXI measurements from near-quadrature at dawn ${ }^{4}$ by measuring over a broad range of scattering angles from 90 deg (dusk) through $180 \mathrm{deg}$ and back to $90 \mathrm{deg}$ (dawn) to derive surface reflectance and cloud/aerosol phase function. Phase angle is defined as the Sun-target-observer angle, with a phase angle of 0 being new Moon and thus full Earth. The phase angles seen from the lunar surface will 
Table 1 EarthShine measures spectral features of astrobiological significance. Note some species overlap spectrally.

\begin{tabular}{ll}
\hline Molecule/feature & \multicolumn{1}{c}{ Wavelengths $(\mu \mathrm{m})$} \\
\hline $\mathrm{H}_{2} \mathrm{O}$ & $0.65,0.72,0.82,0.94,1.12,1.4,2.7,6.5$ (strong) \\
$\mathrm{CO}_{2}$ & $1.44,1.59,4.9,5.3,9.5,10.5$ \\
$\mathrm{O}_{2}$ & $0.63,0.69,0.76$ (strong), 1.27 ( $\mathrm{O}_{2}$ dimer), and $6.5\left(\mathrm{O}_{2}\right.$ dimer) \\
$\mathrm{O}_{3}$ & 0.5 to $0.7,8.8,9.5$ (strong) \\
$\mathrm{CH}_{4}$ & $1.4,1.7,3.4,7.5$ \\
$V^{2}$ getation red edge & 0.7 \\
Glint (best observed at) & 0.7 to $0.9,1.0$ to $1.05,1.3,3$ to 4 \\
Thermal emission & 8 to $9,10.5$ to 12 \\
Soil versus water & 0.85 (high rotational contrast) \\
\hline \hline
\end{tabular}

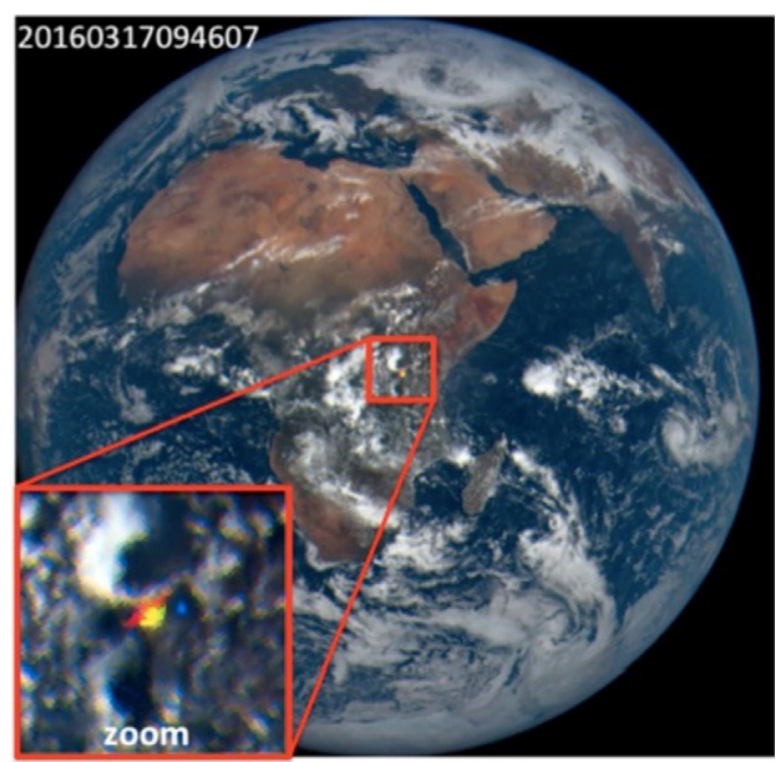

Fig. 3 Sun-glint as a detector of oceans and ice clouds. The specular reflection (glint) from the ocean and from ice crystals in cirrus clouds seen in this DSCOVR/EPIC image will contribute a greater fraction of visible light in crescent phase, seen by EarthShine. EarthShine imaging will test glint as a water indicator.

enable EarthShine to retrieve aerosol and cloud scattering phase functions, monitor vegetation characteristics, and plant physiology, better estimate surface albedo, and image both the day and night Earth.

- Thermal emission phase function. Thermal emission depends upon time of day as well as surface properties that are rotationally modulated. The resulting thermal emission characterizes whether the planetary environment is suitable for organic chemical processes that support life processes.

\subsection{Observing the Global Earth to Inform Earth Climate Studies}

The planet's global radiation budget is critical to interpret both exoplanet atmospheres and Earth's climate, and thus it is remarkable that we do not yet have simultaneous globally 
distributed measurements of Earth's radiated energy. EarthShine measurements will contribute to important Earth Science goals by measuring Earth's radiation budget, accounting for energy exchanged between star (Sun), planet, and space.

The lunar surface offers unique observations not possible from LEO, GEO, or L1 satellites. Radiation budget measurements from the CERES instruments onboard-e.g., NOAA-20, Terra, Aqua, and Suomi National Polar-orbiting Partnership—observe only part of the Earth at specific times; satellites in geosynchronous orbits observe only parts of the Earth and never see the polar regions; GEO satellites $(R / r \sim 6)$, where $R$ is the orbital distance from the center of the Earth, and $r$ is the radius of the Earth, capture phase angle variation but are insensitive to rotational modulation. EarthShine's comparatively distant vantage on the Moon's surface $(R / r \sim 60)$ will enable both of these important components of characterizing unresolved exoplanet climate and surface conditions. The EPIC and NISTAR cameras on the DSCOVER spacecraft at L1 provide only specific, nearly back scattering "full-Earth" direction phase angles. To draw a direct link between L1 observations and Earth's radiation budget, we must observe many scattering angles, not just back scattering. EarthShine observations will provide the missing phase angles. EarthShine will see the whole Earth—including its sunlit and shadowed parts: day, night, and twilight zone-at different phase angles, especially during crescent Earth phases. This complete viewing will help validate Earth (broadband and narrow band) albedo and provide comprehensive (both time and space, compared to LEO) whole-globe monitoring of volcanic and aerosol clouds (smoke and dust), including polar regions not covered by GEO. Further, EarthShine will uniquely inform:

- Global cloud cover estimates. These depend strongly on geometry; measurements from LEO, GEO, and EPIC (from L1) provide different estimates of cloud cover. EarthShine will complement these estimates and provide measurements of daytime variations of global cloud cover.

- Glint reflection (Fig. 3) as a function of solar angle. EarthShine will provide ocean/cloud glint reflection for different phase angles than available in GEO, LEO, and L1. The idea to explore the impact of starlight glints on the detection and characterization of exoplanets goes back to 1993, when Carl Sagan used the Galileo spacecraft's fly-by observation of Earth as an exoplanet control experiment. ${ }^{2}$ Based solely on observations of specular reflection, they deduced that Earth was covered in part by liquid oceans. Their conclusions were based entirely on several Galileo images collected on December 12, 1990, while Galileo was on its way to Jupiter, crossing a line between Sun and Earth. In Ref. 2, they wrote "The Galileo mission constitutes an apparently unique control experiment on the ability of fly-by spacecraft to detect life ...”. Figure 4 shows the spectral behavior of relative enhancements due to cloud glints for ocean and ice clouds based on 10 DSCOVR/EPIC wavelengths. The plot shows that relative enhancements drop at the shortest wavelengths due to the stronger Rayleigh scattering and ozone absorption. This figure also shows that at all wavelengths mean enhancements are about three times stronger over land (cloud ice crystals) than over ocean.

- Vegetated area surface albedo as a function of solar angle. EarthShine will support monitoring vegetation characteristics and plant physiology, estimating bidirectional reflection function.

- Change in radiative properties. EarthShine will characterize the transition from solar insolation dominated regimes in dawn/day to thermal emission domination in dusk/night, with the oblique illumination in dusk/dawn region being sensitive to geographic properties.

Further capitalizing on the complementary nature of EarthShine's Earth Science and exoplanet goals, we will spatially integrate whole-Earth images to simulate spectral light reflection of the Earth as a point source, which will allow us to qualitatively analyze the influence of different surface types, clouds, aerosols, and gases on light signatures. ${ }^{13,19}$ The disc integration involves an ensemble of Earth's disc elements with specific illumination and emission geometries as viewed from a remote vantage. The observing geometries of LEO and GEO orbits are not well suited for selecting an appropriate near-real-time ensemble of these disc elements at a given time and of course spacecraft in such orbits cannot view the entire Earth disk in a single 


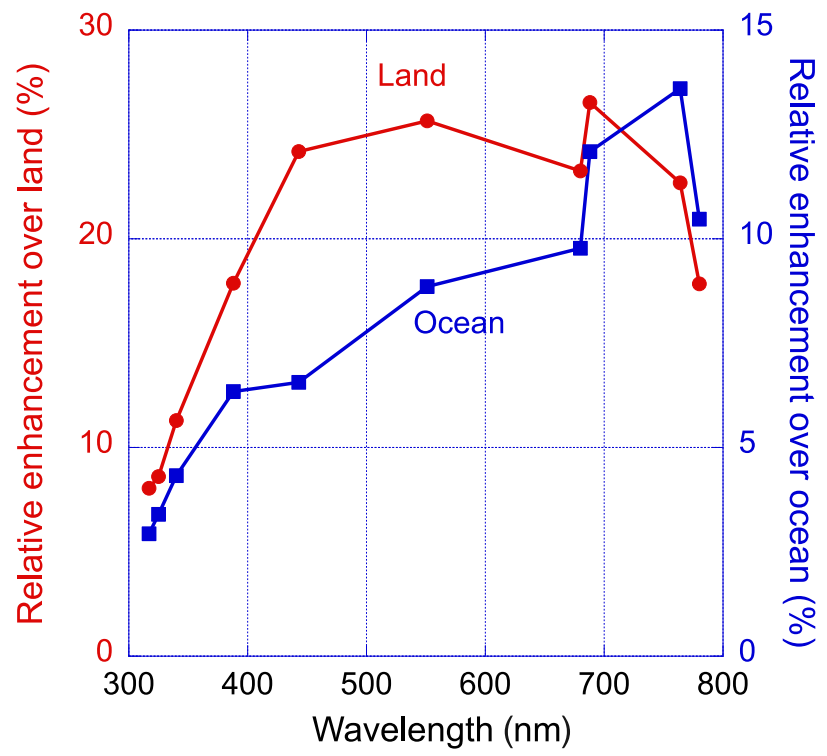

Fig. 4 DSCOVER/EPIC-based wavelength dependence of enhancement caused by specular reflection over land (red) and ocean (blue). Note that 764 and $688 \mathrm{~nm}$ wavelengths are EPIC oxygen absorption $A$ and $B$ bands.

image. The lunar vantage with a range-to-diameter $\sim 60$ deg and 6 deg FOV offers a better representation for this simulation.

EarthShine's two cameras with broad spectral range from visible to IR and day and night coverage (including twilight zone) will allow us to follow dispersion of volcanic or dust clouds for the whole-Earth from daytime to nighttime and back. Since our wavelength coverage includes the $\mathrm{O}_{2}$ A-band $(\sim 760 \mathrm{~nm})$, we can distinguish between two layers of clouds as demonstrated in $\mathrm{Gu}$ et al. ${ }^{19}$ (originating from the combined effect of oxygen absorption and cloud heights).

\subsection{Measurements of Lunar Water in Unique Regions}

Some surface regions such as lunar swirls may offer new options for long-term human presence on the Moon. Lunar swirls are characterized by higher albedo than the surrounding surface, as well as localized magnetic fields, which may have shielded swirls from solar winds and space weathering. Lunar swirls are thought to be of cometary origin (and thus potentially contain significant buried water). Syal and Schult ${ }^{20}$ stated that future missions should test this hypothesis "to fully unravel the secrets of the swirls." Solar wind interaction with the surface is believed to produce the surficial $\mathrm{OH} / \mathrm{H}_{2} \mathrm{O}$ observed pervasively across the Moon. Observations of $\mathrm{H}_{2} \mathrm{O}$ at a lunar swirl with EarthShine's WF-LHR will address whether the "mini-magnetosphere" inhibits the production of $\mathrm{OH} / \mathrm{H}_{2} \mathrm{O}$ at swirls. ${ }^{21-24}$ Deployment in a lunar swirl is not required to achieve the general science goal of observing water vapor in the lunar exosphere from the lunar surface. However, observation of water in a lunar swirl would provide an interesting data point into the origin of these formations and if water is indeed detected, may provide a future landing habitat.

\subsection{Synergistic Benefits of EarthShine Suite}

An essential goal of astrobiology is to detect and understand "other Earths." Thus Earth's own complexity presents the critical test to identify key features describing Earth's climate, properties, and habitability that are detectable at interstellar distance. An "all hands-on deck" approach is essential to capture the complexity of Earth's varied surface of continents, oceans, and ice fields under a transparent Rayleigh-scattering atmosphere containing water as gas, liquid, and solid simultaneously, hosting planetwide (technological) life, with a rocky satellite in the mix now known to show detectable hydration. The EarthShine suite will sample reflected light, 
as well as thermal self-emission, to characterize global spectroscopic chemical signatures, rotational modulation, phase curves, and energy balance of Earth, creating a rich phase space that can be explored to identify the most critical features for future reconnaissance, when space observatories will capture the first unresolved low-spectral resolution measurements of terrestrial exoplanets. EarthShine will also be sensitive to measuring water vapor at the lunar deployment site, characterizing a critical feature that may be a source of confusion in unresolved measurements of planet-moon systems. Although EarthShine data will include spatially resolved spectral imaging of Earth that would be impossible for the first-generation detection systems currently envisioned, these data will be needed to constrain what deductions can and cannot be substantiated from future spectroscopy and spectrophotometry. EarthShine will assess the discovery space of measurement targets that are not yet recognized, as well as features of habitability such as the vegetation red edge that have already been proposed, creating a laboratory to pursue investigating possible habitable worlds.

\subsection{EarthShine in Context}

Previous studies of Earth as an exoplanet have measured "Earthshine," defined as sunlight that has been reflected from the dayside Earth onto the dark (shadowed) side of the Moon. Earthshine measured from ground-based telescopes involves the additional step of measuring the light reflected from the dark side of the Moon back to observers on Earth. ${ }^{14,25,26}$ Turnbull et al., ${ }^{14}$ collected integrated Earth spectra reflected off the dark Moon and concluded that strong water signatures could be detected that would clearly imply habitability and further advocated that the NIR would be a powerful waveband for undertaking searches for habitability on exoplanets. A complementary transmission spectrum of Earth reflected off the Moon during a lunar eclipse ${ }^{27}$ showed that several biologically relevant atmospheric features are significantly more prominent in transmission than in reflection. Measuring Earth's spectrum from the Moon would provide far more robust measurements than measuring Earthshine from Earth's surface. In ground observations, spectra pass through Earth's atmosphere three times, complicating correction for telluric absorption and lunar reflectance. ${ }^{4}$ Further, the lunar vantage would allow us to collect spectral features inaccessible from the ground (Table 1). The idea of using the Earth as our best-studied habitable planet, to inform exoplanet research, is not new. From space, Galileo fly-bys in the early to mid 1990s collected images of Earth at a variety of wavelengths and illumination phases (Table 2). Sagan et al. ${ }^{2}$ deduced that Earth was teeming with water and identified signatures that together are strongly suggestive of life, including abundant $\mathrm{O}_{2}$, the VRE, and atmospheric $\mathrm{CH}_{4}$ in thermodynamic equilibrium. The Mars Global Surveyor ${ }^{3}$ observed Earth during cruise and obtained the first known thermal IR spectra of the Earth's whole disk, identifying key spectral features in Earth's atmosphere. Spacecraft in near-Earth orbit have obtained a vast amount of Earth imaging data (e.g., Hearty et al.), ${ }^{15}$ although synthesizing the data to produce full-Earth images is challenging (and demonstrates the value of "distant-Earth" observations that can validate calibration and imaging techniques, rendering these data useful for exoplanet studies.)

In 2008, as part of the Extrasolar Planet Observations and Characterization (EPOCh/EPOXI) program, the NASA Deep Impact mission observed the "unresolved" Earth as an exoplanet proxy, collecting hourly images in several Vis/NIR photometric bands for three separate 24-h periods at illumination phases from $66 \%$ to $77 \%$, using both the high-resolution visible imager and the high-resolution imaging infrared spectrometer. The data imply that sophisticated observers in nearby extrasolar systems could differentiate between surface areas covered by oceans versus continents on Earth, ${ }^{4,6-8}$ suggesting that we can pursue the same types of studies when characterizing small rocky exoplanets with future direct-imaging flagship missions. Similarly, the LCROSS mission collected shorter data sets on three separate occasions in 2009 at three widely separated Earth phases, detecting ocean glint and ozone signatures, in addition to further calibrating models. ${ }^{9}$ Further, Kane et al. ${ }^{10}$ used multispectral imaging data from EPIC/DSCOVR,${ }^{11}$ degraded to a pixel resolution comparable to that of our most optimistic future exoplanet observations and demonstrated that time variations in the data could be used to determine Earth's rotation, obliquity, and atmospheric albedo—crucial observables expected to determine surface conditions on remote exoplanets. Numerous authors have proposed innovative uses of DISCOVR data to consider Earth as an exoplanet. ${ }^{14,15,25-32}$

J. Astron. Telesc. Instrum. Syst. 014003-8 Jan-Mar 2022 • Vol. 8(1) 
Boyd et al.: EarthShine: Observing our world as an exoplanet from the surface of the Moon

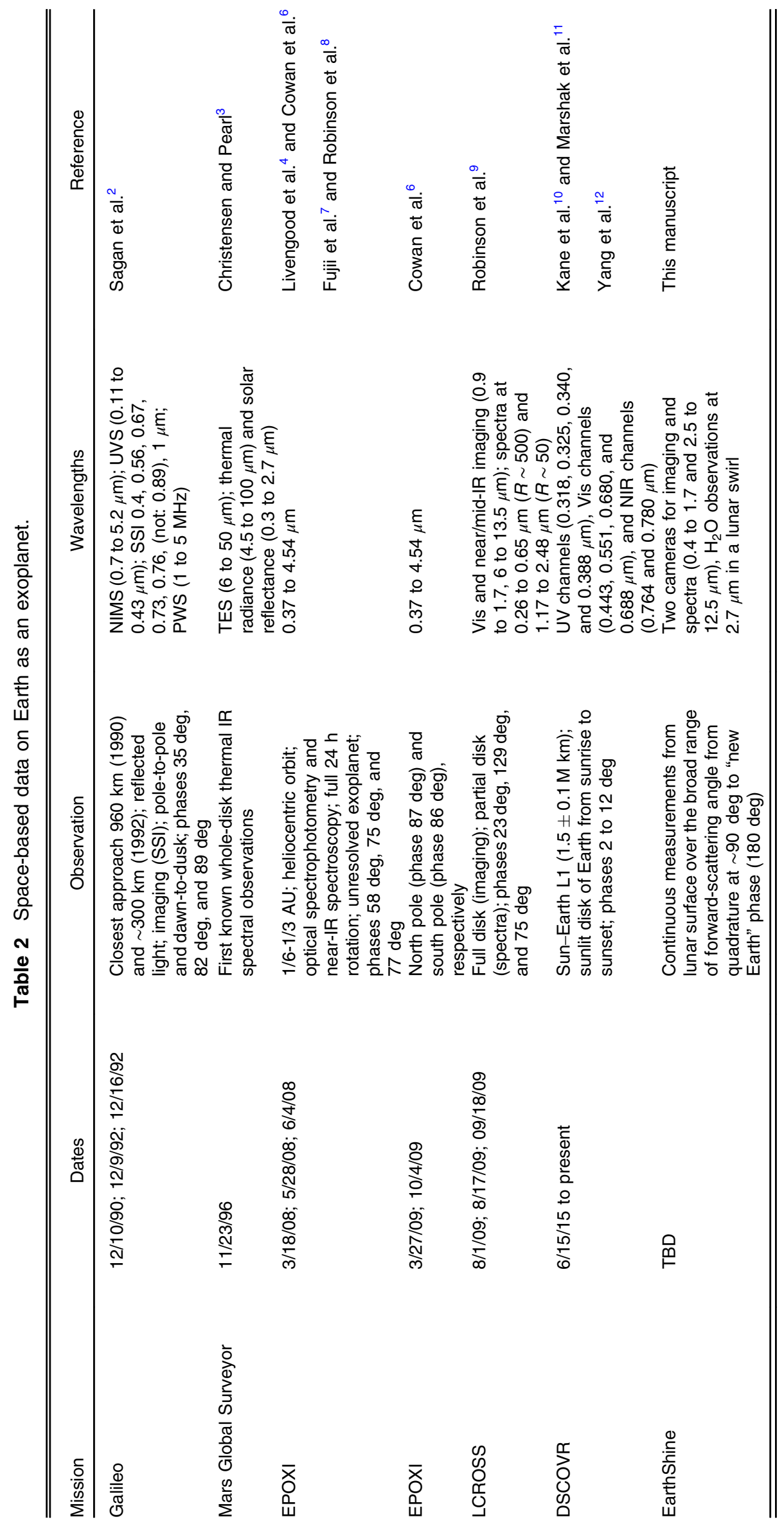




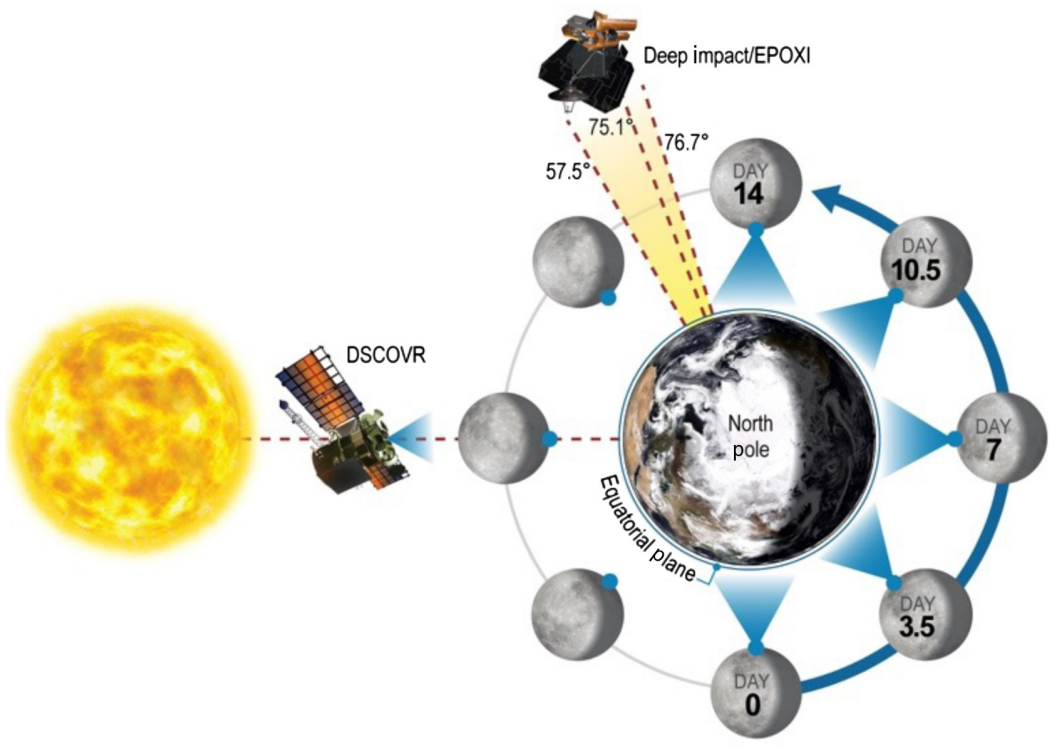

Fig. 5 The power of EarthShine. Observations would begin at day 0, viewing the waning gibbous Earth at dusk. Lunar noon, day 7, views the "new Earth" at mission mid-point. The payload would go dormant near day 14, with a waxing gibbous Earth at dawn. By comparison, DSCOVR can view only "full Earth," while EPOXI viewed only a modest range of phase angle on the dawn side.

EarthShine will collect observations of Earth from space at illumination phases not yet observed (Fig. 5), with a consistent set of instruments, simultaneously spanning wavelength ranges of interest (Fig. 6). EarthShine's comprehensive data set will enable us to identify distinctive characteristics of an Earth-like planet and life and to quantify how these features change with time.

To establish realistic, disc-integrated (or barely spatially resolved), spectroscopic representation of an Earth-like exoplanet, we need observations of Earth's entire disk from a remote vantage. Such a database is critical in the interpretation of future observations of an exoEarth. This requires whole-disk spectroscopic observations of Earth acquired at various phase angles. Currently, our continuous whole-disk observations of Earth have primarily come from the

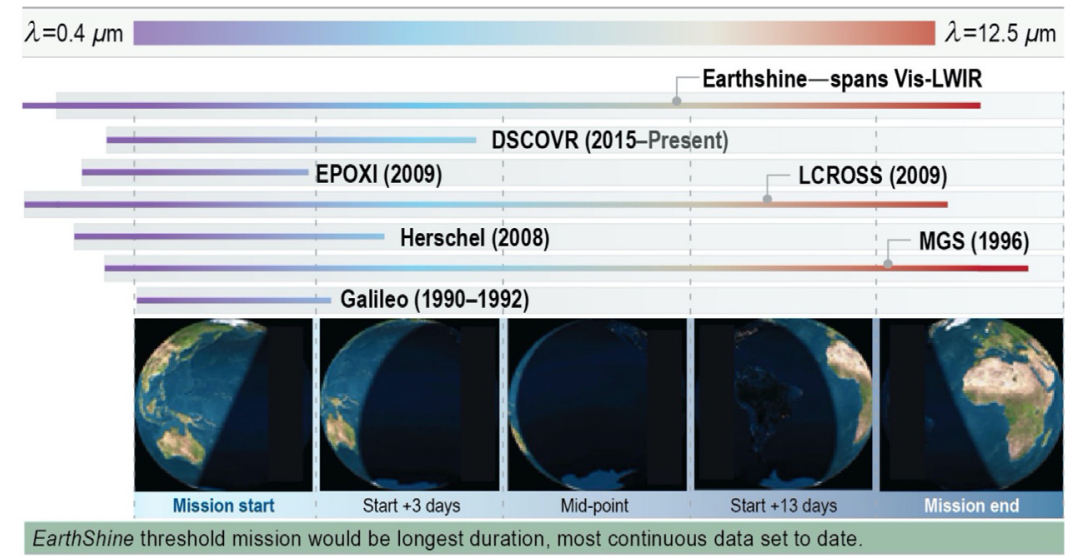

Fig. 6 EarthShine tests our ability to detect and interpret terrestrial exoplanets. EarthShine will observe phase angles not well assessed before, measuring signatures of critical species $\left(\mathrm{H}_{2} \mathrm{O}, \mathrm{CO}_{2}, \mathrm{CH}_{4}, \mathrm{O}_{3}\right.$, and $\left.\mathrm{O}_{2}\right)$ in Earth's reflectance spectrum from the visible to NIR range, over forward-scattering angles from $90 \mathrm{deg}$ (dusk) to "new Earth" at $180 \mathrm{deg}$ (midnight) to $90 \mathrm{deg}$ (dawn), providing continuous coverage of Earth's varying reflectance phase function for the first time. Horizontal lines represent wavelength ranges of the missions. EarthShine will be the first empirical test for IR methods to investigate exoplanet chemistry and climate. 
DSCOVR and EPOXI missions. However, EPOXI had limited phase sampling and time duration. DSCOVR has observations over a longer timeline but only covers the Earth at full phase and neither transit spectroscopy nor direct imaging will access exoplanets at this phase. EarthShine will fill this critical void in understanding the spectroscopic presentation of an Earth-like exoplanet with observations of the Earth's full disk at multiple phases, dramatically improving the database of observations and enabling future exoplanet spectroscopy mission design.

We will relate the observed spectroscopic signatures collected with EarthShine to physical processes acting on the planet using accurate radiative transfer modeling and retrieval methods. By employing these methods used to infer properties of exoplanets, we can better understand the relationship between measured signatures on our planet and the significance of actual bio/geo-markers on exoplanets. Over the last decades, modeling and retrieval capabilities for interpreting Earth's data have matured tremendously, ${ }^{33,34}$ with Robinson and Reinhard ${ }^{35}$ summarizing the latest methods when interpreting Earth's data as an exoplanet. For our forward models and retrieval analysis, we will employ the planetary spectrum generator ( $\mathrm{PSG},{ }^{36,37}$ developed to be broad in scope to capture the latest methods and techniques from astronomy, Earth, and Planetary Science communities. PSG integrates the latest advancements in 3D scattering radiative-transfer modeling ${ }^{38}$ and has access to a comprehensive spectroscopy library, including billions of absorption lines from $>1200$ species, aerosol scattering models for 104 species, collision induced opacity sources for more than 20 pairs, and surface properties for 2000 materials.

Considering the whole-Earth disk nature of EarthShine observations, modeling and interpreting the data require accurately capturing $3 \mathrm{D}$ global information. The recently developed module for PSG, GlobES (Global Exoplanetary Spectra), is specifically tailored to allow ingest of 3D climatological data and retrieve/compute information from the emergent spectra. GlobES was recently demonstrated in an application to TESS's first Earth-size Habitable-zone World, TOI$700 \mathrm{~d} .{ }^{39}$ The comprehensive data collected with EarthShine will further advance and validate these methods, following an approach similar to Robinson et al., ${ }^{8}$ by generating an ensemble of synthetic spectra with PSG to compare with the time-variable spectral and brightness variations collected across the wide EarthShine spectral bands. This will lead to a more robust understanding of model uncertainties, while also facilitating the investigation of seasonal and temporal variations in biosignature components. ${ }^{40}$

\subsection{Unique Advances Enabled by EarthShine}

EarthShine is the empirical test needed by the exoplanet community for modeling remote observations of terrestrial planets detected at other stars. EarthShine will cover a broad range of phase angle and wavelength in both reflected light and self-emission, covering the phase angle range of $90 \mathrm{deg}$ through $180 \mathrm{deg}$, from dusk to midnight to dawn. No prior direct measurements exist of the whole Earth in this range of phase angle, which covers half the range of possible remote measurements. DSCOVR/EPIC has observed Earth extensively in UV, visible, and NIR wavelengths at 2 deg to 12 deg phase angle, and Deep Impact/EPOXI covered a limited range of 57 deg to 75 deg phase angle in visible to NIR wavelengths (Fig. 5). Combined with EPIC and EPOXI reflectance data, EarthShine will define Earth's reflectance spectrum and phase curve over the full range from back-scatter to forward-scatter and compare dawn to dusk to search for any observable asymmetry. EarthShine will obtain the first ever thermal-IR global measurements over a range of phase angle and with sensitivity to rotational modulation, defining spectroscopic sensitivity required to detect biosignature gases (e.g., $\mathrm{CH}_{4}$ and $\mathrm{O}_{3}$ ) and habitable environments. These measurements will set requirements for exoplanet measurement capabilities in future flagships, whether using optical coronagraphy, IR interferometry, or direct imaging.

The EarthShine threshold mission is illustrated in Fig. 5, which is compatible with the currently available resources on today's CLPS providers. Even a short 9- to 14-day mission would yield a significant improvement over the data available from previous missions listed in Table 2 . As future power and temperature-control resources improve for the lunar surface payloads, it should be possible to extend the mission duration design to multiple lunar days, and survival through the lunar night, further enhancing the science reach of EarthShine by allowing for 

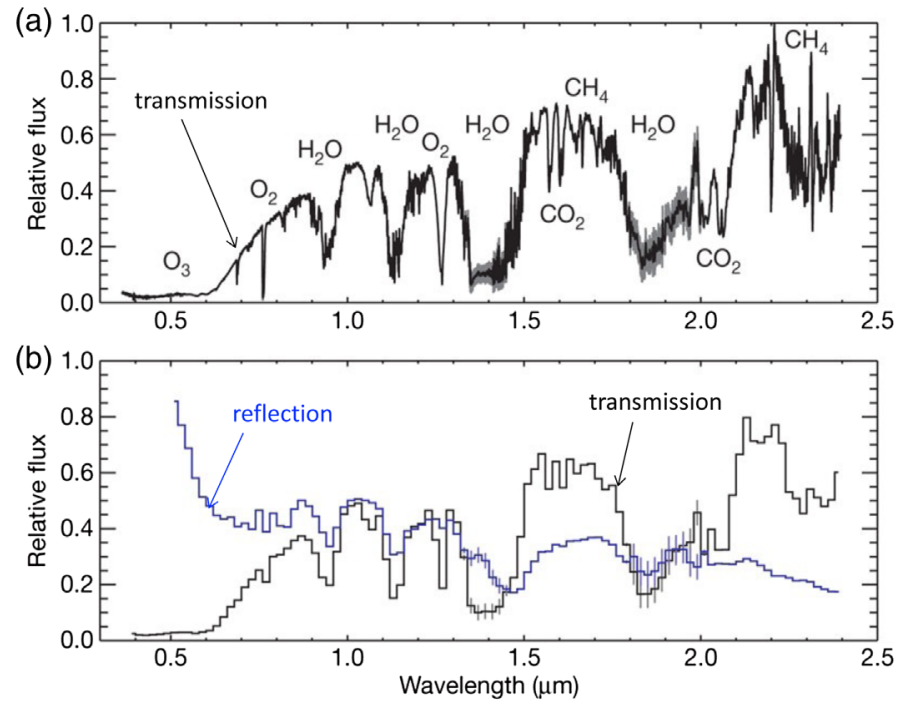

Fig. 7 Observations during lunar eclipse reveal fingerprints of a habitable world. ${ }^{27}$ Visible-to-nearIR light reflecting off the fully eclipsed Moon passes through a ring of Earth's atmosphere. (a), (b) The observed transmission spectrum (black) is analogous to what a distant observer watching Earth transit in front of the Sun would see, while measurements at other lunar phases are analogous to a directly imaged reflection spectrum (blue).

observations of Earth phases analogous to those future missions will collect to investigate potentially habitable exoplanets. In the near-term, alternative landing sites on the lunar near-side may shift the mission toward somewhat greater access to the dusk-side or dawn-side range of phase angle. For example, a site at $\sim 60 \mathrm{deg}$ Eastern longitude on the Moon (toward the celestial West limb) would see sunrise $\sim 5$ days before gibbous-phase Earth on the dusk side and would see sunset $\sim 5$ days before reaching gibbous phase on the dawn side. Offsetting in the opposite direction in longitude would achieve later sunrise and later sunset, with more access to the dawn side.

\subsubsection{Transmission and reflection spectra}

The Earth is the best-studied habitable planet to inform observations from future exoplanet missions. Its transmission spectrum is a proxy for Earth observations during a primary transit as seen from beyond the Solar System, while its reflection spectrum is a proxy for the observations of the Earth as an exoplanet by direct observation (after removal of the Sun's spectral features).

As an illustration, Fig. 7(a) taken from Ref. 27 shows the Earth's transmission spectrum, with some of the major atmospheric constituents marked. Below it, we show a comparison between the Earth's transmission (black) and reflection (blue) spectra. Both spectra have been degraded to a resolution of $0.02 \mu \mathrm{m}$ (to emulate spectra from exoplanets) and normalized at the same flux value at around $1.2 \mu \mathrm{m}$. It is clear from this figure that the reflection spectrum shows increased Rayleigh reflectance in the blue. It is also striking how most of the molecular spectral bands are weaker, some even non-existent, in the reflection spectrum.

Variations due to large-scale exoplanetary features such as continents and oceans will be contained in perhaps a single pixel. Disentangling the various components that give rise to time variability in the signal will be a challenge. To assess the limits of this issue, our image data will be further deconvolved to $3 \times 3$ pixel resolution following the approach used by Kane et al. ${ }^{10}$ on EPIC data (see Fig. 8).

\subsubsection{Solar eclipses}

From the lunar surface, EarthShine will have a ringside seat for a number of eclipses of the Sun by the Earth. As examples of the cadence of solar eclipses as seen from the Moon, the next total 

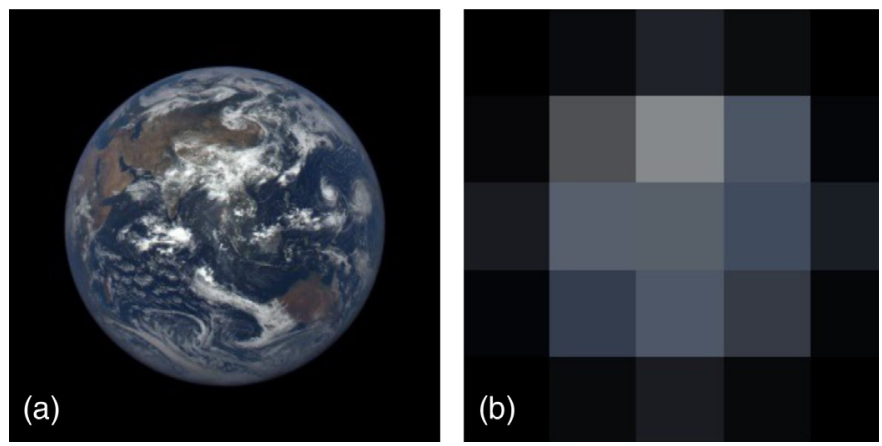

Fig. 8 (a) Earth as seen from Earth/Sun L1, using the DSCOVR-EPIC camera. (b) The same image degraded to $3 \times 3$ pixel resolution, analogous to expectations of future exoplanet observations. A single, or a few, pixels may contain all the available information on the habitability of an exoplanet. Credit: NOAA/NASA/Kane et al., $2017 .^{10}$

solar eclipses will occur on March 14, 2025, September 7, 2025, March 3, 2026, August 28, 2026, December 31, 2028, and December 2029, with four further total eclipses between 2032 April and 2033 October. In addition, between 2024 and 2034 there will also be 15 partial and penumbral eclipses, where the Sun will transition through the field of view of our instruments while being only partially blocked by the Earth. (Note that due to the larger size of the Earth, many lunar eclipses listed as "partial" as viewed from the Earth will actually be total solar eclipses as seen from some points on the lunar surface, looking in the opposite direction.) The maximum duration of a solar eclipse as viewed from the Moon is $5.5 \mathrm{~h}$. Eclipse observations could test the detectability of technosignatures and set limits on what might be observable in exoplanet systems.

\subsubsection{Earth occultations of solar system planets and bright stars}

As seen from the Moon, the Earth occults bright stars and other Solar System planets on a regular basis. With EarthShine, we may obtain data as these objects transition "through" (behind) the Earth's atmosphere. These data can be converted into exoplanet-like transit spectra, as described by Robinson et al. ${ }^{41}$ and demonstrated by Macdonald and Cowan. ${ }^{42}$ Measurements of Earth occultations of stars with a variety of stellar types would yield further detail on Earth's atmosphere and allow us to build a database of star-planet observations to compare with exoplanet transmission spectroscopy.

Using the Stellarium planetarium software ${ }^{43}$ we have simulated EarthShine's view of the Earth and the celestial bodies that the Earth will occult. Based on an analysis of data from the 8-year period 2023 January 1 through 2030 December 31, we find that EarthShine will observe 2 to 8 occultations per month of Solar System planets or stars with a visual magnitude brighter than 3. These stars include Spica (visual magnitude 0.95), Antares (1.05), Alcyone (1.2), Regulus (1.35), Beta Tau (1.65), Rho Sgr (2.05), Delta Sco (2.35), Alpha2 Lib (2.75), Lambda Sgr (2.8), and Pi Sgr (2.85). Of the planets, Mercury ( -1.06 to 4.78$)$, Venus $(-4.13$ to -3.56 ), Mars ( -0.48 to 2.05$)$, Jupiter ( -1.65 to -1.31$)$, Saturn (1.05 to 1.38$)$, and Uranus ( 5.8) are also regularly occulted, with their broad range of apparent magnitudes depending on their distance and degree of solar illumination. Typically, the passage of a celestial body through the Earth's atmosphere (as seen from the Moon) lasts 5 to $8 \mathrm{~min}$. Such observations are aspirational and not guaranteed, and their technical feasibility will be addressed in Sec. 3, following the descriptions of the CHEILS and EPyC-IR instruments.

\subsection{Lunar Site Selection}

The science achievable with the EarthShine instrument suite depends very little on the choice of landing site, although longitude offset will affect the details of the beginning and ending Earth phase available for observation. Provided that the site is located on the Moon's near side, and that

J. Astron. Telesc. Instrum. Syst. $\quad$ 014003-13 Jan-Mar 2022 • Vol. 8(1) 
EarthShine is deployed with an uninterrupted view of the Earth, our instruments can achieve all of the science goals described in this paper. As seen from the Moon, the Earth's position in the sky follows an elliptical path with a semimajor axis of $\sim 15 \mathrm{deg}$, passing north and south of the ecliptic by $\sim 5$ deg. Thus the requirement to have the Earth constantly in the EarthShine field of view rules out sites above $\sim 85$ deg in lunar latitude.

As seen from the Moon, the Earth's disk subtends an angle of $\sim 1.9 \mathrm{deg}$, so the details of the eclipses and occultations described above vary in only minor ways regardless of whether the chosen site is equatorial or at high latitude. The thermal load on the payload will, of course, depend strongly on the lunar latitude, as described fully in Sec. 4.

\subsection{Additional Potential Secondary Science Objectives}

\subsubsection{Quantification of lunar impact dust accumulation rates on lander's solar panels}

The Moon possesses a permanent tenuous ejecta-driven asymmetric dust cloud. ${ }^{44}$ Models for the meteoroid bombardment of the Moon are able to reproduce the shape of this tenuous cloud, but there is a several order of magnitude disagreement in the cloud density. ${ }^{45}$ So far, only a handful of in situ measurements of the deposition of lunar dust have been made, all by the Apollo missions. ${ }^{46}$ All Apollo missions took serious measures to mitigate the degradation of equipment and experiments by the presence of lunar dust, however, the dust accretion caused a wide range of operational problems such as overheating and subsequent failure of experiments from Apollo 11 through Apollo $17 .{ }^{47}$ It is essential for any future lunar exploration to quantify the deposition of lunar dust and to reconcile the global lunar dust models and various observations.

There are two main non-anthropogenic (not caused by humans) mechanisms that drive lunar dust accumulation on human space exploration timescales: (1) meteoroid impacts striking the lunar surface and ejecting $\sim 100 \times$ their mass ${ }^{48,49}$ and (2) electrostatic transport at the lunar terminator. ${ }^{50-52}$ Meteoroids continuously bombard the entire lunar surface in a stochastic manner, whereas the electrostatic transport at the lunar terminator happens once per 14 days. ${ }^{49}$ These two effects act on different timescales and should be separable. While different models exist, there is no widely accepted consensus for both (1) and (2).

Human activity locally disturbs fine regolith on the lunar surface. ${ }^{53}$ The local disturbance sets free a large amount of dust. During the lunar sunrise, these locally disturbed regolith particles are hit by energetic UV and soft x-ray photons that cause positive surface charging. ${ }^{51}$ This results in a sudden electrostatic repulsion of dust particles causing them to levitate until a balance between the gravitational force and the electrostatic repulsion is established. The height of this effect was observed in situ and estimated to be $1 \mathrm{~m}$ above the surface. ${ }^{54}$ However, recent experiments show that the electrostatic dust lifting is reciprocally proportional to the particle radius, depends on the particle shape, and shows a broad range of launch angles. ${ }^{55}$ Some of these charged particles merge via cohesive forces into clumps. ${ }^{56}$ These clumps increase in mass, which decreases their levitating height during the next lunar sunrise. Consequently, the pool of fine regolith initially supplied by anthropogenic disturbances depletes with successive lunations. ${ }^{57}$ This phenomenon is most important during the early phases after the mission landing and should decrease in importance after several lunations.

Solar cells provide continuous voltage measurements that allow to indirectly measure the lunar dust deposition over long periods of time. The higher number of solar cells will enable more precise measurements of the deposition of lunar dust than were possible in the Apollo 12, 14, and 15 missions, that contained only small, matchbox-sized silicon solar cells with blue filters and heavily shielded against radiation with a focus on dust detection. ${ }^{57}$ Furthermore, a mounted camera could repeatedly observe the lunar dust deposition on various portions of the lander to determine the cohesion and accumulation of the lunar dust on different materials and areas with different slopes. Expected bandwidth is low, since the data cadence does not need to be high and the data amount is $\sim 1000$ numbers per measurement. The cadence of visual observations of the lander/solar panels will depend on the detailed operations plan; but observations on a timescale of hours to a few days would be a good strategy. 

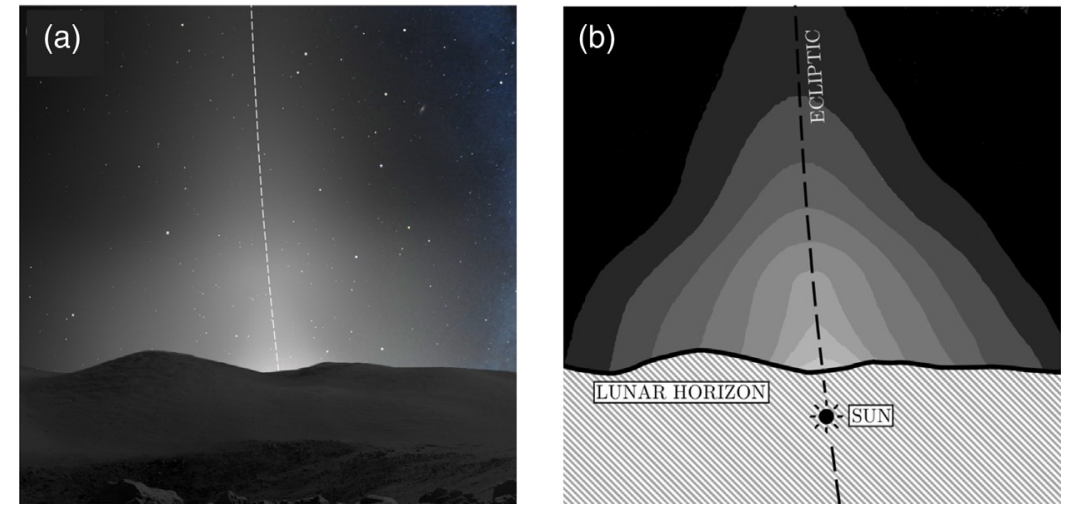

Fig. 9 Artist's impression of the zodiacal light observation from the lunar surface. (a) Simulated zodiacal light observation from a lunar lander pointed to the west. The zodiacal light appears as a triangular white glow. The Sun is several degrees below the lunar horizon allowing us to probe the fine structure of the zodiacal light in the inner solar system. The white dashed line represents the ecliptic which represents an approximate line of symmetry of the zodiacal cloud in the inner solar system. (b) The sketch version of (a). The lunar horizon is represented by a solid black line while the dark lunar surface is shown as a shaded area. The Sun is located several degrees under the lunar horizon and is represented by a black symbol. The ecliptic is shown as a black dashed line. The zodiacal light is shown as a gradient of grayscale approximately resembling our zerothorder estimate for the visual flux. Notes: (a) was created using the Stellarium program (zodiacal light and celestial sphere) and Apollo 17 panorama (lunar surface). (b) is a heavily processed version of (a).

\subsubsection{Inner solar system zodiacal dust observations in visible wavelengths}

Although the innermost parts of the zodiacal cloud (ZC) are studied by solar oriented missions (spacecraft traveling toward the Sun $;^{58}$ spacecraft orbiting at $1 \mathrm{AU}^{59}$ ), the global shape of the inner parts of the ZC has been the subject of only a few studies. The global shape of the ZC is a key component of our understanding the structure and origin of the dust in the Solar System, thus providing important insight into Solar System space weather and exosolar dust debris disks. Using the lunar surface/horizon as an effective Sun shade, we can in better detail analyze the extent of the ZC in visible light using the EarthShine star tracker similar to that on the Clementine spacecraft ${ }^{60}$ (see Fig. 9).

The global shape of the ZC is driven by various processes: (1) the production of dust in asteroids and comets, ${ }^{61}(2)$ the dynamics of the dust driven by gravity and radiative processes,${ }^{62}$ and (3) the elimination of dust in various sinks. ${ }^{63}$ Each model of the $\mathrm{ZC}$ strives to match the global density profile of the dust, however, such observations are rare. ${ }^{60}$ The $\mathrm{ZC}$ is composed of dust particles originating from vastly different sources: main-belt, ${ }^{64}$ Jupiter-family comets, ${ }^{65}$ and long-period comets. ${ }^{66,67}$ Each of these sources provides a unique signature in the $\mathrm{ZC}$ and their mixing ratios are not well understood. ${ }^{68}$ The Clementine spacecraft 6 -week survey provides the only global inner solar system snapshot of the $\mathrm{ZC}$ in visible wavelengths. ${ }^{61}$ The Clementine mosaic suffers from several drawbacks: (1) it extends only from 3 deg to 30 deg away from the Sun, (2) its detector is rather primitive compared to today's standards, and (3) due to various exposure times and only a six-week window, the data product suffers from poorly defined uncertainties and pollution from bright Venus' signature.

We propose to reproduce the Clementine mosaic in much greater detail and longer data coverage using the EarthShine star tracker cameras. Thanks to our enhanced knowledge of the inner solar system dust dynamics ${ }^{45}$ and previous observations by various spacecrafts and especially Clementine, ${ }^{53}$ we can fine tune the star tracker exposure time to better survey the inner ZC. To overcome the limited data bandwidth, the data will be analyzed in situ and only the final data products will be sent back to Earth. The original Clementine data set consists of only seven observations, thus there is a vast room for improvement to be made by Earthshine. 


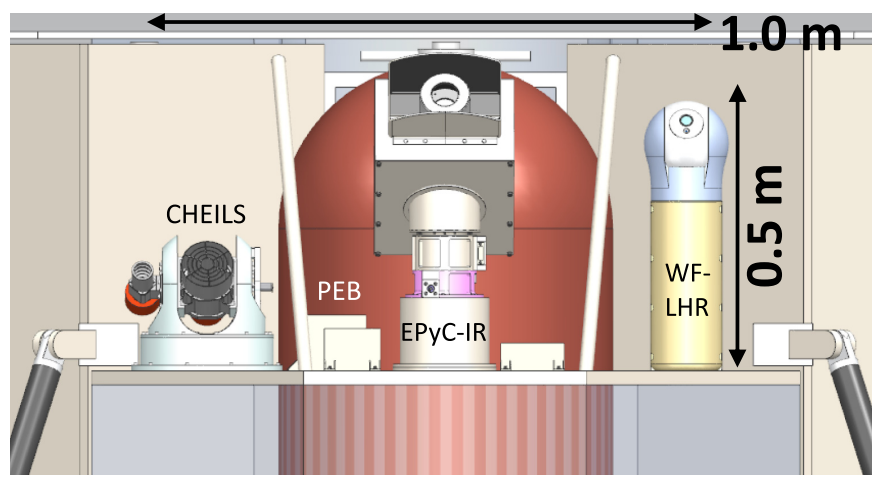

Fig. 10 EarthShine is a compact suite of three instruments that can fit a wide range of CLPS lander options. The deck of the Astrobotic Peregrine lander, as shown here, is the smallest accommodation for CHEILS, EPyC-IR, WF-LHR, and a common Payload Electronics Box.

\section{EarthShine Instrument Suite}

The EarthShine suite (shown in Fig. 10) consists of three instruments that operate in the visible, infrared, and thermal spectrums. CHEILS is an LLNL developed visible near-infrared/ shortwave-infrared (VNIR/SWIR) grating spectrometer that observes $\mathrm{O}_{2}, \mathrm{O}_{3}, \mathrm{H}_{2} \mathrm{O}, \mathrm{CO}_{2}$, and $\mathrm{CH}_{4}$ in the Earth's atmosphere from 0.4 to $1.7 \mu \mathrm{m}$. The WF-LHR is a GSFC developed passive laser heterodyne radiometer that observes $\mathrm{H}_{2} \mathrm{O}$ in the lunar exosphere at sunrise and sunset at picometer resolution between 2.6668 and $2.6674 \mu \mathrm{m}$. EPyC-IR also developed at GSFC is a compact hyperspectral imaging camera spanning the 2.5 - to $12.5-\mu$ m medium-wavelength IR (MWIR) to long-wavelength IR (LWIR) spectral range and observes $\mathrm{H}_{2} \mathrm{O}, \mathrm{OH}$, and mineral signatures and infrared emission from Earth. Detailed descriptions of these instruments follow.

\subsection{CHEILS}

Developed at LLNL, CHEILS is an Earth-imaging grating spectrometer with a fused silica monolithic telescope fore-optic that is pointed at the Earth using a two-axis gimbal mechanism and ultimately focused on a COTS Sony IMX990 back-illuminated InGaAs detector housed in an Aval Imaging ABA-013VIR camera. A schematic of CHEILS is shown in Fig. 11 with key specifications summarized in Table 3.

The compact, high-performance, monolithic telescope [Fig. 12(a)] was originally developed for deployment on small satellites and aircraft where size and weight come at a premium. We refer to them as monolithic telescopes because they are constructed from a single block of highpurity fused silica, which provides intrinsic mechanical/thermal stability (negligible thermal expansion) when operating in extreme environments. The spectrometer disperses incoming light

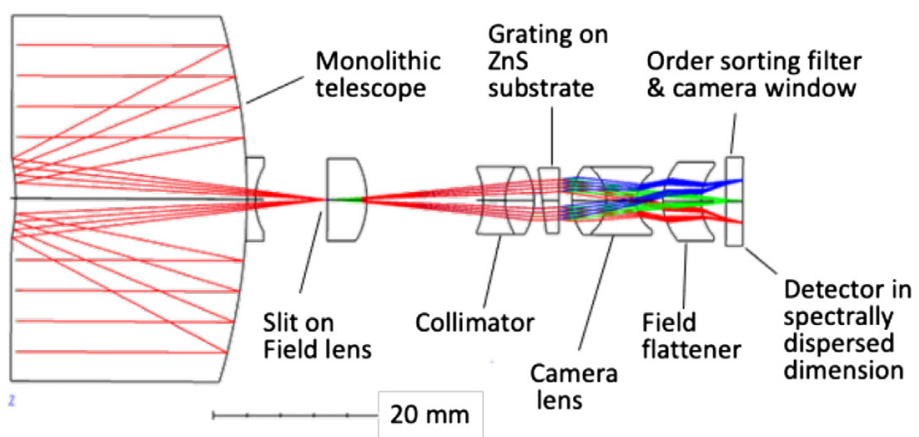

Fig. 11 CHEILS schematic. Elements are cemented together and mounted in an Invar housing. Monolithic construction provides high environmental robustness, no postlaunch and landing alignment needed. 
Table 3 CHEILS telescope, spectrometer, and detector descriptions.

\begin{tabular}{ll}
\hline \hline Telescope & \multicolumn{1}{c}{ Spectrometer } \\
\hline - FOV at max image field: $2.5 \mathrm{deg}$ & - 400 to $1750 \mathrm{~nm}$ spectral coverage \\
- Clear aperture: $44 \mathrm{~mm}$ & - 1024 spectral bins (1.32 nm/bin) \\
- Focal length: $147 \mathrm{~mm}, \mathrm{f} / 4.0$ & - 1280 spatial pixels along slit \\
- Entry face to slit: $38 \mathrm{~mm}$ & - Average RMS spot size of $3 \mu \mathrm{m}$ \\
- Diffraction limited & - Slit width equal to pixel pitch \\
- Spectral range: 400 to $1750 \mathrm{~nm}$ & - All refractive elements \\
- Obscuration: $7.3 \%$ by area & - LLNL ruled dual-blaze grating on ZnS substrate \\
- Material: fused silica & - Order sorting filter at FPA \\
- Field corrector glued to monolith & - Optics train length = $101 \mathrm{~mm}$ \\
Detector & \\
- Sony IMX990 back-thinned InGaAs & - High QE from 400 to $1700 \mathrm{~nm}$ \\
\hline - Small $5 \mu \mathrm{m}$ pixels enable compact sensor & - $1280 \times 1024$ format \\
\hline \hline
\end{tabular}

using an in-house ruled dual-blaze grating on a $\mathrm{ZnS}$ substrate [JWST near-infrared imager and slitless spectrograph (NIRISS) grating ruled in ZnSe shown in Fig. 12(b)].

Pointing of CHEILS is accomplished with a gimbal that is being designed by LLNL for an International Space Station (ISS) mission with a delivery set for late 2021 and a launch in January 2023. The mechanism utilizes two Empire Magnetics motors, each driving a pulley system.

During operation, CHEILS step-stares across the Earth's disk at a rate of 7.0 arc sec /step with a maximum scan time of $\sim 20 \mathrm{~min}$. Each scan produces a data cube with native spatial dimensions of 1280 cross-track $\times 1024$ along-track with each spatial location having an associated 1024 bin spectrum covering a 400- to $1750-\mathrm{nm}$ spectral region. Integration time is set to produce $1 / 2$ to $2 / 3$ maximum well-filling for high-albedo locations (Fig. 13). Depending on the scan frequency and uplink availability/capacity, onboard data processing algorithms will reduce data cube volume by rebinning spatially and/or spectrally, cropping data cube to pixel's above detection threshold, and applying data compression for uplink.

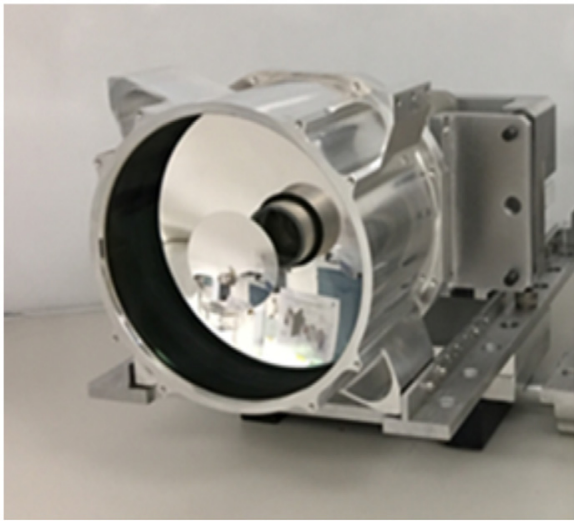

(a)

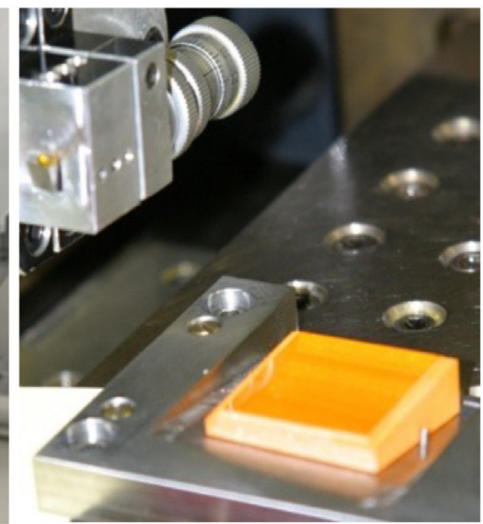

(b)

Fig. 12 (a) Monolithic telescope mounted in Invar housing and (b) LLNL ruling JWST NIRISS grism. 

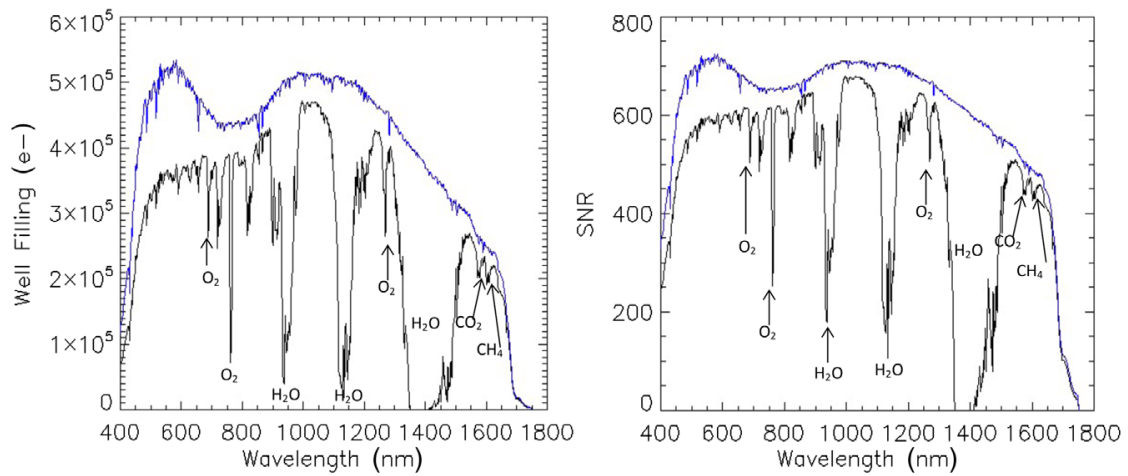

Fig. 13 (a) Calculated well-filling and (b) SNR values for Lambertian reflection of solar top-of-theatmosphere radiance and from Earth's surface with 0.9 albedo. Integration time/frame $=1 \mathrm{~s}$.

Observations of solar eclipses (Sec. 2.6.2) and stellar and planetary occultations by the Earth's atmosphere (Sec. 2.6.3) would place additional requirements on the CHEILS hardware. CHEILS cannot directly observe the Sun without damage unless we place a neutral density filter in the optical path ahead of the focal plane array (FPA). This filter mechanism is not included in the instrument diagrams provided here to reduce complexity but can be included at a later date. Viewing the Earth while in total eclipse would be feasible with the current instrument configuration. Viewing partial eclipses, or at other times when the Sun is in or close to the FOV, would require the neutral density filter.

The CHEILS telescope will have a baffle in front to mitigate stray light. Measurements of similar baffles show a $1 \times 10^{-12}$ stray light background per Earthshine pixel when the light source is $5 \mathrm{deg}$ outside the field, and $1 \times 10^{-14}$ at $30 \mathrm{deg}$. As a $1 \times 10^{-12}$ intensity reduction is equivalent to $\sim 30$ visual magnitudes and the brightness difference between the Sun and the Earth is $\sim 11$ magnitudes, a solar angle limit of $5 \mathrm{deg}$ would likely be sufficient for operational purposes.

The feasibility of the stellar eclipses by the atmosphere will depend critically upon the details of the payload operations, which have yet to be specified for the Artemis program. CHEILS is designed to sweep over the $\sim 2$ deg-wide disk of the Earth with 0.25 deg of cross-track pointing error, accommodated by an oversized slit. For a stellar occultation measurement, the 2.5-deg slit projection should be more than adequate to accommodate any cross-track pointing error. The along-track accuracy, however, would present a challenge: the slit width will subtend $\sim 10$ arc sec, so without dithering the required pointing accuracy would need to be $\sim 2$ arc sec. This may be achievable later in the mission, once the sensor pointing offsets have been characterized well enough.

\subsection{WF-LHR}

The WF-LHR is based on the GSFC team's earlier laser heterodyne radiometer developments (shown in Fig. 14) that include an Earth-based ground instrument that measures $\mathrm{CH}_{4}$ and $\mathrm{CO}_{2}$ in the atmospheric column (mini-LHR), and an occultation-viewing CubeSat (MiniCarb) designed to observe $\mathrm{CH}_{4}, \mathrm{CO}_{2}$, and $\mathrm{H}_{2} \mathrm{O}$ from an LEO. ${ }^{69-73}$ Similar in design, the WF-LHR will be solar pointing and observe $\mathrm{H}_{2} \mathrm{O}$ in the lunar exosphere at sunrise and sunset when the pathlength is closest to the lunar surface and has the highest density of molecules.

A schematic of the WF-LHR is shown in Fig. 15. A fiber-coupled telescope mounted to a sun tracker delivers light to mix with a distributed feedback laser centered at $\sim 2.67 \mu \mathrm{m}$. The interference between frequencies enables a resolving power of 160,000 with signal-to-noise ratio $(\mathrm{SNR})>300$ for $1 \mathrm{~s}$ integration time/data point. The laser scans across these features and the resulting beat signal is collected in the radio frequency and converted to mole fraction.

The WF-LHR uses a GSFC-developed tracker for solar pointing. The LHR collimator is housed within the tracker base as are two stepper motors for control of zenith and azimuth and control electronics. The pointing accuracy is better than $0.06 \mathrm{deg}$ and it operates autonomously. 


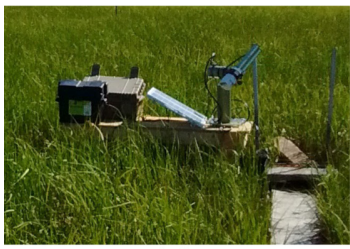

(a)

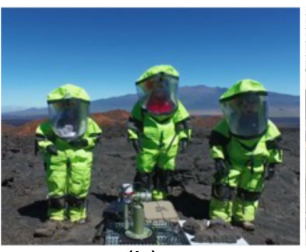

(b)

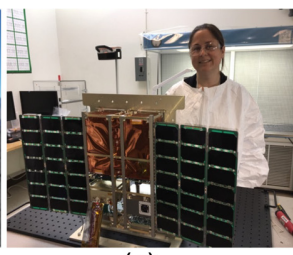

(c)

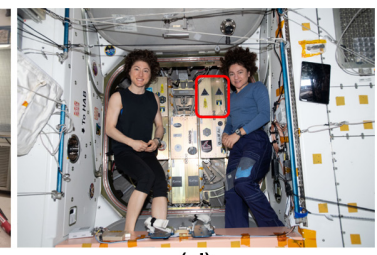

(d)

Fig. 14 LHR developments: (a) ground LHR in Alaska measuring $\mathrm{CH}_{4}$ over permafrost; (b) Hawai'i Space Exploration Analog and Simulation team members using a ground LHR during a long-duration Mars simulation program; (c) the joint GSFC/LLNL CubeSat MiniCarb at integration; and (d) MiniCarb on the ISS prior to deployment (payload at upper left, indicated in red).

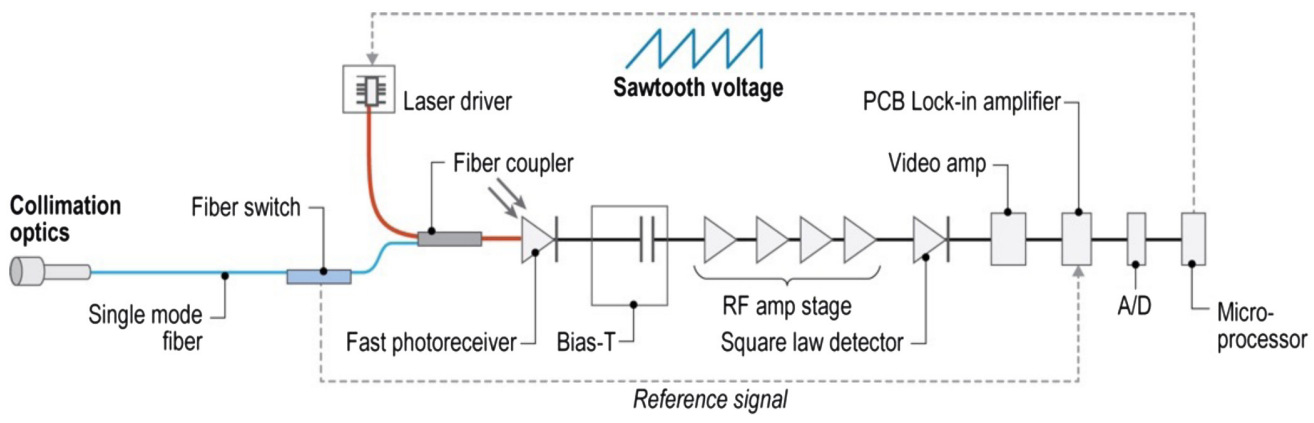

Fig. 15 The WF-LHR design leverages postage-stamp-sized distributive feedback lasers from the telecommunications industry to produce a compact and cost-effective package.

The tracker dimensions are (508-mm height, $159 \times 166-\mathrm{mm}$ width), and approximate mass is $4.5 \mathrm{~kg}$.

Figure 16(a) shows sample data from column observations from one of our ground mini-LHR instruments operating at a permafrost site in Alaska with comparisons to a co-located flux tower that is observing gas concentrations at the surface. Data (blue) are analyzed using the retrieval tool of Goddard's PSG. ${ }^{38,74,75}$ The fit from this retrieval is shown in orange. Figure 16(b) shows projected observations of water vapor in the lunar exosphere. In this PSG simulation, the transmittance is modeled for a solar-pointing WF-LHR that is deployed in Reiner Gamma assuming a hydrostatic equilibrium atmosphere, a surface pressure of $1 \times 10^{-3} \mathrm{~Pa}$, molecular weight of $40 \mathrm{~g} / \mathrm{mol}$, atmospheric temperature of $300 \mathrm{~K}$, surface temperature of $130 \mathrm{~K}$, albedo of 0.136 , and emissivity of 0.864 . During operations, the WF-LHR points at the sun and observes
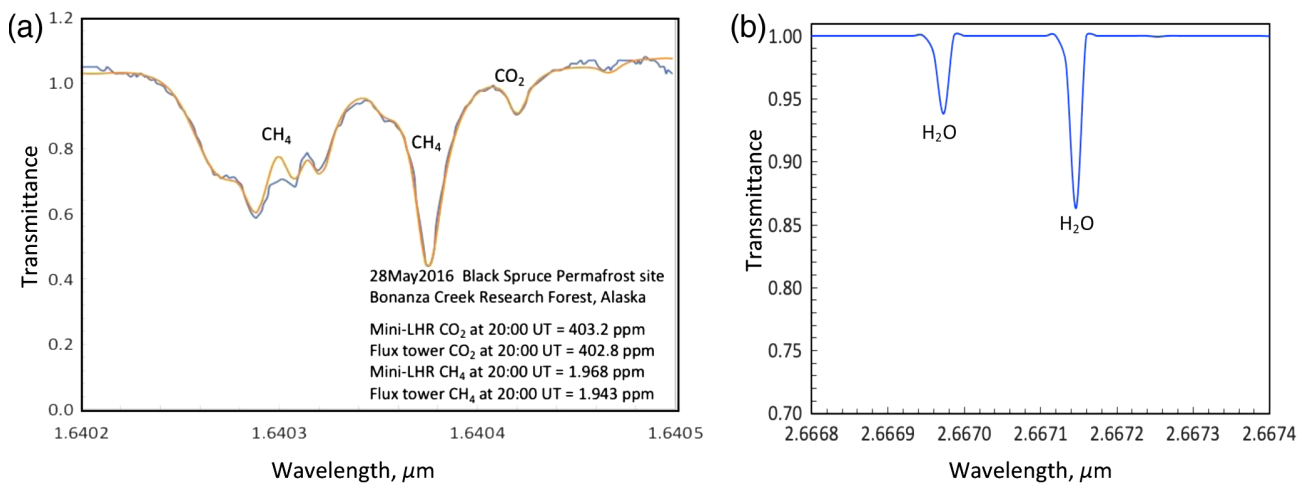

Fig. 16 (a) Heritage from mini-LHR ground observations on Earth. Half-hour column data products show good agreement with surface observations at the site. Data are shown in blue and the PSG retrieval fit is shown in orange. (b) Simulated transmittance of $\mathrm{H}_{2} \mathrm{O}$ vapor lines in the lunar exosphere modeled with the PSG for the WF-LHR (Villanueva et al. 2018, ${ }^{36}$ Edwards $\left.1992^{34}\right)$. 
absorption from water vapor in the lunar exosphere at sunrise and sunset to maximize airmass. The WF-LHR scans continuously from 2.6669 to $2.6674 \mu \mathrm{m}$ with 60 points per scan, each scan taking approximately $1 \mathrm{~min}$ assuming a 1-s integration time per data point.

\subsection{EPYC-IR}

The EPyC-IR is a GSFC-developed compact hyperspectral imaging camera spanning the 2.5- to 12.5- $\mu \mathrm{m}$ MWIR to LWIR spectral range. This spectral range contains habitability diagnostic spectral features of an exoEarth $\left(\mathrm{H}_{2} \mathrm{O}, \mathrm{CH}_{4}, \mathrm{CO}_{2}, \mathrm{O}_{3}, \mathrm{~N}_{2} \mathrm{O}, \mathrm{CO}, \mathrm{SO}_{2}\right.$, etc.), existence of atmospheric stratification, radiative balance between parent star insolation and thermal emission, surface properties regulating emissivity, and thermal structure (Fig. 17). EPyC-IR consists of the type-II strained layer superlattice (SLS) sensor, linear variable filter (LVF) assembly, Ricor cryocooler, and a catadioptric telescope. The heart of the focal plane imaging system is the sensor chip assembly consisting of the $1024 \times 1024$ format $18 \mu \mathrm{m}$ pixel pitch SLS InAsSb/InAs sensor chip hybridized to an FLIR Indigo ISC0404 readout-integrated circuit. The pixel spectral response of the SLS technology is extremely uniform and temporally stable. The focal plane assembly is cooled to $\sim 70 \mathrm{~K}$ by a miniature Ricor K508N Stirling tactical cryocooler.

A commercial $200 \mathrm{~mm}$ focal length, $f / 2$ catadioptric telescope from StingRay Optics also brackets the same spectral range and matches the $18 \mu \mathrm{m}$ pixel scale. EPyC-IR is supported by an azimuthal scan and elevation-positioning mechanism leveraged from another instrument.

Compact thermal imager (CTI) ${ }^{76}$ the heritage instrument, successfully observed Earth system scenes, including ocean, forest canopy, clouds, savanna and deserts, cryosphere regions, and transients such as biomass combustion (e.g., Fig. 17) from the ISS in 2019. EPyC-IR leverages

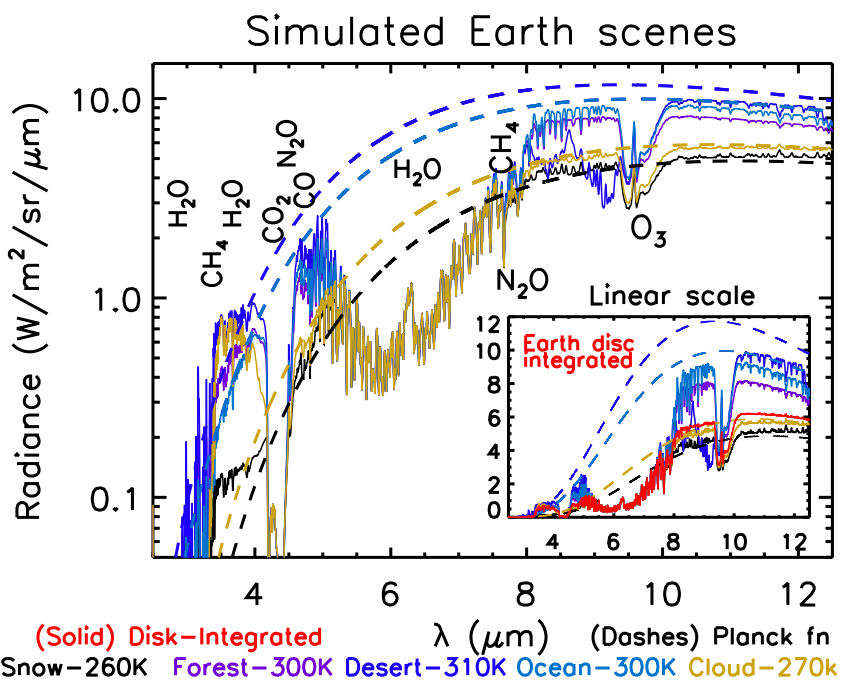

Fig. 17 EPyC-IR spectroscopy complements CHEILS reflected light measurement. Earth's nadirviewed spectrum from 2.5 to $12.5 \mu \mathrm{m}$ (modeled) is bracketed by EPyC-IR and includes reflected light for $<3 \mu \mathrm{m}$ (opaque due to water opacity) transitioning to thermal emission at longer wavelengths. Critical gases diagnostic of the habitable Earth-like atmosphere are clear in this range $-\mathrm{H}_{2} \mathrm{O}, \mathrm{CO}_{2}, \mathrm{O}_{3}$, and $\mathrm{CH}_{4}$. These signatures can be measured in exoplanets only from space, and thus methods can be tested only in space observations of Earth. Balance between pure blackbody functions (dotted lines for 260, 270, 300, and $310 \mathrm{~K}$ ) and calculated atmospheric emission (snow, forest, desert, ocean, and cloud) constrain composition and thermal structure. A cloudy planet presents a colder scene for 3.5 to $4.2 \mu \mathrm{m}$ and $>10 \mu \mathrm{m}$; the $300-\mathrm{K}$ vegetation spectrum is dominated by lower emissivity than a 300-K ocean or desert in the LWIR region and is manifest as the dimmest of these three scenarios in this range. The global (disc integrated) spectrum includes contributions from terrains with unique spectral signatures such as visible suppression by savanna vegetation, low-albedo over ocean, and reduced emission due to ice absorption at 3.5 to $4 \mu \mathrm{m}$ over snow. Models for crescent-phase spectroscopy change the weighting of features and must be tested empirically. 
(a)

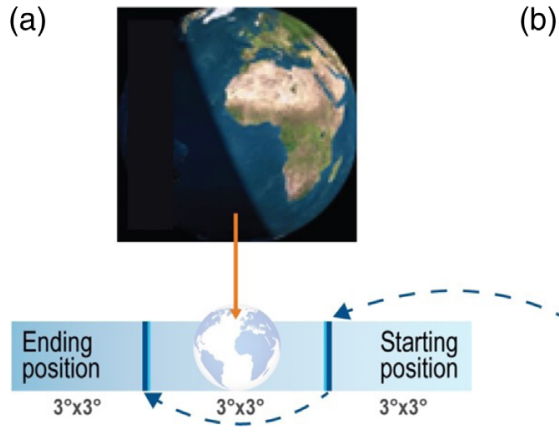

(b)

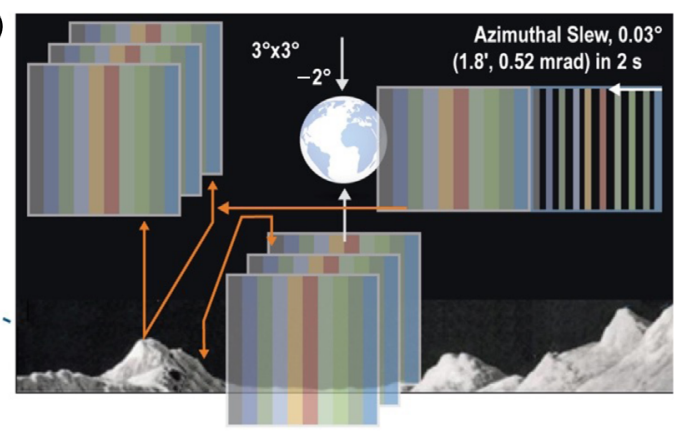

Fig. 18 EPyC-IR hyperspectral maps across Earth. EPyC-IR sweeps an LVF in step-and-stare operation to acquire a hyperspectral data cube. Three contiguous 3 deg $\times 3$ deg fields-of-view are required to sample the 2-deg diameter Earth (a) in all wavelength bands to reconstruct wavelength band-sequenced imaging frames. (b) Each colored column of the 3 deg $\times 3$ deg FOV represents a wavelength band on the LVF and each column samples a different spatial region. In the stepand-stare operation, each column will gradually sweep adjacent spatial regions. Thus a sweep between the starting and ending field-of-view positions (a) will produce one data cube of the center field of view with complete spectral sampling. Spectral resolving power and/or spatial resolution are selectable to meet data transmission limitations.

design features from prior developments: electronics and imaging core (CTI), LVFs (OSIRISREx/OVIRS), and optics and scan mechanism (other mission instruments).

To acquire the hyperspectral data cube of Earth disc for a given phase, EPyC-IR will use a gimbal mechanism, which will enable step-and-stare frame acquisition bracketing the disc with all spectral bands. The LVF results in spectral columns sampling different spatial regions; the mechanism then steps the 3-deg EPyC-IR field of view by 0.03 deg (corresponding to the $1 \%$ filter FWHM or 102 pixels of the 1024 span).

During step-stare operations, EPyC-IR frames are captured at a regular $50 \mathrm{~Hz}$ rate with an integration time of $\sim 1 \mathrm{~ms} /$ frame, acquiring 1-ms exposures at $20 \mathrm{~ms}$ frame intervals. Fifty frames are coadded and stored in the instrument control electronics (Fig. 18). Each column of 102 pixels projected to sky plane samples a spatial slice in the spectral band of the column, with adjacent column sampling a different spatial region at the next wavelength; the mechanism then steps EPyC-IR by $0.03 \mathrm{deg}$ (corresponding to the 1\% filter FWHM or 102 pixels of the 1024 span). Repeated step-and-stare operations capture the Earth's disk (100 steps of $0.03 \mathrm{deg}$ ) with a set of 100 spectral bands as the EPyC-IR FOV transitions between the starting and ending FOV configuration (Fig. 18).

EPyC-IR is currently optimized for Earth disc measurements rather than stellar occultation observations through the Earth's atmosphere, but such observations should still be possible. Efficacy will be established through models to establish limiting stellar brightness as a function of lander heat rejection capabilities. For example, additional cooling of telescope optics elements will allow for reduction in thermal emission with a resulting improvement in the detection sensitivity of stellar point sources.

For the case of a total solar eclipse, a fully unilluminated Earth allows for greater sensitivity. Since totality within the umbral region will be longer than that typical of solar eclipses on Earth, we envision pointing the telescope to observe the Earth's limb to observe stellar occultations. An additional mitigation under consideration is to include a long-pass optical filter $(>2.4 \mu \mathrm{m})$ to block the visible-to-SWIR solar insolation which may cause damage to optical coatings. The optimum position for such a filter is in front of the primary mirror (catadioptric telescope design) and requires a broadband antireflection coating (2.5 to $12.5 \mu \mathrm{m})$ to minimize optical losses.

The ability to discriminate molecular vibrational signatures of water vapor from $\mathrm{OH}$ is unambiguous in the MWIR/LWIR spectral region. The $2.7-\mu \mathrm{m}$ vapor phase stretch mode ( $\sim 3 \mu \mathrm{m}$ in water ice and hydroxyl species) is common to both $\mathrm{H}_{2} \mathrm{O}$ and $\mathrm{OH}$; in comparison, the $\sim 6-\mu \mathrm{m}$ vibration bending mode is unique to molecular $\mathrm{H}_{2} \mathrm{O}$ and is a prime water proxy in a surface water rich planet such as Earth. In addition to thermometry of the planet, other signatures relevant to an exoEarth analog in this spectral region include $\mathrm{CO}_{2}, \mathrm{O}_{3}$, and $\mathrm{CH}_{4}$. Infrared self-emission is an 
effective way to characterize terrestrial exoplanets due to the detectability of these diagnostic atmospheric species at habitable temperatures as well as constraining theoretical estimates for equilibrium temperature. The relatively favorable contrast ratio between the primary star and planetary thermal emission, $\sim 1: 10^{4}$ compared to $\sim 1: 10^{9}$ at visible wavelength, has previously led to developing designs for large-scale infrared interferometers that may yet be selected for deployment. Exploring this spectral range is essential to evaluate the viability of the various spectral intervals available.

Characterization by EarthShine in MWIR and LWIR will enable identification of spectroscopic wavelengths and optimum bandwidths for high spatial resolution chronographic systems optimized for capturing images of an exoEarth in biosignature-indicative spectral bands. Other applications of the characterization include transit studies of exoplanets in diagnostic wavelengths identified by EPyC-IR to identify and quantify composition and thermal structure.

\section{Thermal Design}

The thermal solution for EarthShine is driven by the power profile and operating temperature limits of the payload and by the latitude of the landing site on the Moon, which will determine the lunar surface temperature and the path of the Sun across the sky during the surface mission. The EarthShine payload thermal design is primarily passive, using high-efficiency multilayer insulation (MLI) blankets and optical solar reflector (OSR) radiator coatings to manage heat input and rejection, with the lander providing the primary radiation service for the payload. Supplemental survival heaters will prevent temperatures from dropping too low, particularly during the cruise to the Moon and (if permitted by the chosen lunar lander) during the lunar night.

Initially, we will consider the most demanding cases (Table 4): a landing site close to the lunar equator, where the solar radiation during lunar day is maximized at lunar noon (hot case), and the cruise phase during transport to the Moon (cold case). For an equatorial landing spot, the lunar temperature at orbit noon is expected to be $\sim 400 \mathrm{~K}$ based on the worst-case environmental parameters.

During the cruise phase, the EarthShine suite could look extensively at deep space, at a temperature of $2.3 \mathrm{~K}$. For the hot case, thermal conduction is assumed to meet a $+45^{\circ} \mathrm{C}$ base temperature at our mounting interface for the electronics. In addition, we assume that the lander (or associated power source) will be able to provide $123 \mathrm{~W}$ of heat rejection from the payload. Upon selection of a specific lander, we will conduct an integrated thermal analysis to ensure compatibility. For $123 \mathrm{~W}$ and an OSR radiator system with degraded properties, the required radiator area would be $\sim 0.51 \mathrm{~m}^{2}$. Should the lander not be able to support the interface, our payload itself will need to determine mounting options a radiator of this size.

For a landing site at higher latitude, the required radiator area required to deal with the heat of lunar day might be considerably reduced, as indicated by Figs. 19 and 20.

For the opposite extreme of the cold case, during transfer orbit, the thermal design will utilize survival heaters (Kapton thin film) with bimetallic thermostats in order to maintain a $-20^{\circ} \mathrm{C}$ non-operating temperature during launch and coast phases. During the lunar transit cruise, the EarthShine $0.5-\mathrm{m}^{2}$ radiator will need 60 to $90 \mathrm{~W}$ of survival heater power to maintain this

Table 4 Hot and cold case environments.

\begin{tabular}{lcc}
\hline \hline & Hot & Cold \\
\hline Solar $\left(\mathrm{W} / \mathrm{m}^{2}\right)$ & 1420 & 1280 \\
Albedo factor & 0.13 & 0.06 \\
IR (at subsolar C1) $\left(\mathrm{W} / \mathrm{m}^{2}\right)$ & $1420 \times(1-0.06)=1335$ & $1280 \times(1-0.13)=1114$ \\
$\mathrm{IR}\left(\right.$ cold side C2) $\left(\mathrm{W} / \mathrm{m}^{2}\right)$ & 5 & 5 \\
\hline \hline
\end{tabular}


Boyd et al.: EarthShine: Observing our world as an exoplanet from the surface of the Moon

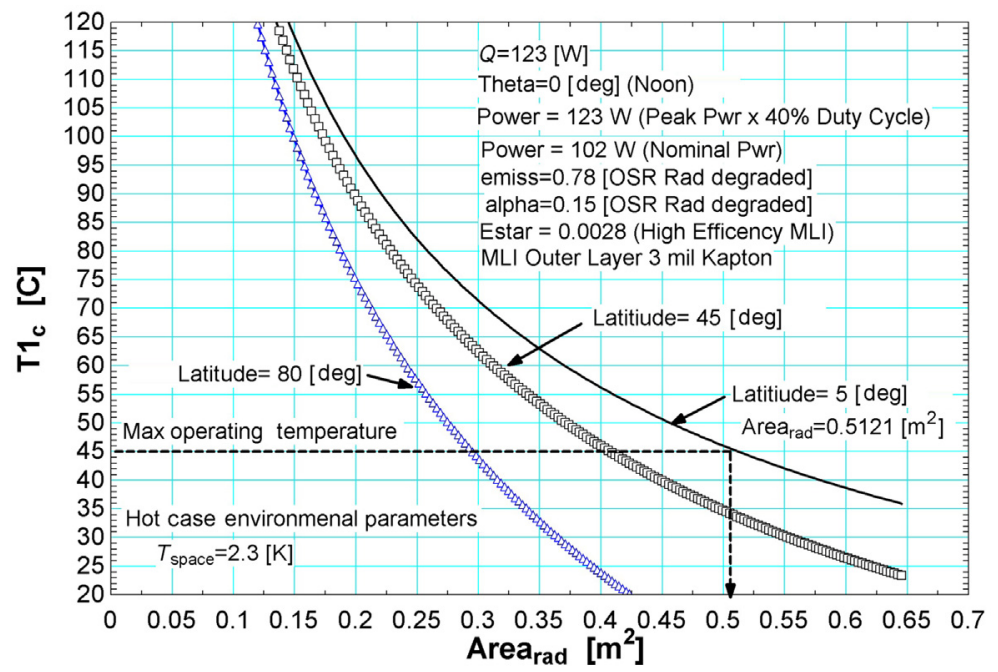

Fig. 19 EarthShine keeps its cool. Shown is temperature sensitivity to power dissipation and radiator size and latitude. For a site close to the lunar equator, EarthShine nominal power dissipation of $102 \mathrm{~W}$ (peak $123 \mathrm{~W}$ ) is balanced against maximum operating temperature of $45^{\circ} \mathrm{C}(318 \mathrm{~K})$ by radiative cooling from surface area $0.51 \mathrm{~m}^{2}$. Curves for latitudes of $45 \mathrm{deg}$ and $80 \mathrm{deg}$ are also shown.

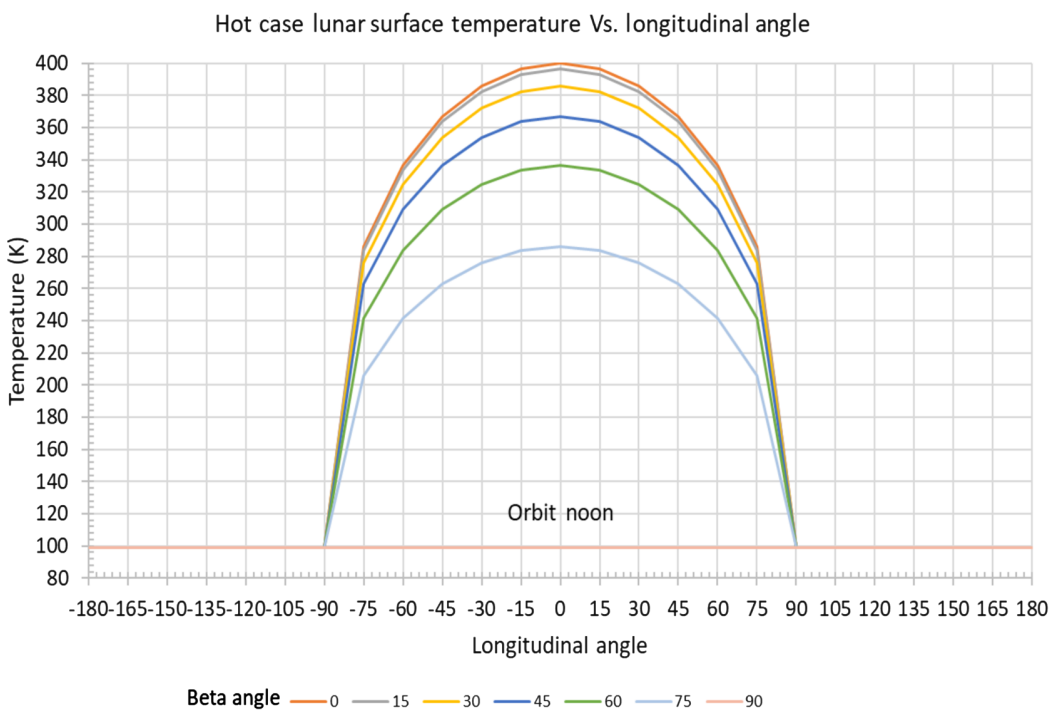

Fig. 20 EarthShine can stand the heat. Lunar surface temperature is symmetric about a maximum of $400 \mathrm{~K}$ at noon, increasing steeply from $\sim 100 \mathrm{~K}$ at dawn to $>280 \mathrm{~K}$ at the $\sim 07: 00$ local solar time position in which EarthShine will land at low latitude and diminishes steeply from 17:00 to dusk. Surface survival will require cooling to maintain operating temperature, after Vasavada et al. ${ }^{77}$

$-20^{\circ} \mathrm{C}$ survival temperature, assuming a radiator emissivity of 0.78 (OSR) and a clear view to space.

On the lunar surface, the EarthShine payload will use high-efficiency MLI with a 3-mil Kapton outer layer on all exposed surfaces to minimize the influence of environmental hot/cold extremes. We will employ a gimbal elevation shield to ensure no exposure of the inside of the gimbals. Depending upon the specifications of the lander and the EarthShine payload's location on it, other design options might be available-for example, the lander itself might provide a degree of shading at some times during the lunar day. These options will be explored with an integrated thermal analysis. 


\section{Data Processing, Analysis, and Archiving}

The data gathered by EarthShine will be scientifically compelling and visually spectacular, and a foundational principle of the EarthShine project is to ensure that they are made broadly available to the astrophysics, Earth Science, and Planetary Science communities, as well as the Citizen Science community and the general public. We plan to supply EarthShine data in easily accessible formats, and relevant software required for analysis in open-source formats and venues. Our team has a broad and deep track record of generating and widely disseminating science results, data products, software, and associated information, and we will bring this experience to bear to ensure that scientists and the public reap full benefits from EarthShine.

Data products from the three instruments are separate but complementary. CHEILS is a VNIR/SWIR imaging spectrometer that observes $\mathrm{O}_{2}, \mathrm{O}_{3}, \mathrm{H}_{2} \mathrm{O}, \mathrm{CO}_{2}$, and $\mathrm{CH}_{4}$ and provides spatial-spectral-temporal data from scanning the Earth, with data products to include images, spectra, and time series. The raw data form an event cube with 1024 spectral bins and a $256 \times$ 256 spectral resolution across Earth's disk. The instrument electronics bin these data prior to downlink.

The primary data product will be images of the full Earth, varying with phase and rotation, which will have huge public appeal in addition to their scientific value. Additional products will include spatially resolved and calibrated spectra of the Earth, plus any additional specialized products that we will generate to maximize the scientific and outreach appeal of any total or partial eclipses observed, occultations of bright stars and Solar System planets by the Earth's atmosphere, or other targets of opportunity.

The WF-LHR is a solar-pointing laser heterodyne radiometer that observes water vapor in the lunar exosphere through the collection of ultra-high-resolution spectra. These spectra are of very high quality but small in data volume, and a limited number of scans since sunrise and sunset are the targeted observation windows. These solar transitions provide the longest path through the lunar exosphere and consequently the largest number of potential water molecules. We anticipate a default time resolution for WF-LHR scans of $\sim 60 \mathrm{~s}$. Each scan produces data that will construct a single spectrum.

EPyC-IR is a thermal spectrometer, to observe infrared active $\mathrm{H}_{2} \mathrm{O}, \mathrm{CO}_{2}, \mathrm{CH}_{4}$, and $\mathrm{O}_{3}$ atmospheric molecular fingerprints superimposed on terrain signatures in Earth's thermal emission spectrum. The hyperspectral Earth maps created by the step-and-stare process described above will be rebinned using a methodology similar to that of the CHEILS instrument, and the data products will include images, spectra, time series, and other associated data products.

We anticipate that EarthShine unprocessed level 0 data will flow into a Science Operations Center and pass through a processing pipeline to perform necessary calibrations and verify data quality. Data products will be released in standard FITS formats, which have a substantial multimission heritage in the astronomy and Planetary Science communities, and for which publicly available analysis software already exists. These files will also be interoperable within the Planetary Science PDS4 format, in primary use to store and distribute solar, lunar, and planetary image data.

Our goal is to share the EarthShine data with the world in real time (with the lowest possible latency). The community outreach impact of being able to see a real-time image of the Earth from the Moon, and watch this view changing on a timescale of hours, is considerable. Whether or not this is feasible depends on technical details of the CLPS (or other) lander selected, and NASA's Artemis data infrastructure, but our overall goals would be to: (a) make Earth images available in seconds to minutes or with minimum possible delay; (b) make all spectra available once the data are processed and calibrations applied, plus a short interval for validation/verification ( $\sim$ hours/days); and (c) make the full data cubes available on completion of more detailed checking (days). There will be no proprietary time for EarthShine data, and these data will reside in established NASA astrophysics and planetary data archives.

In an hour, the Earth rotates by $15 \mathrm{deg}$, thus preserving a minimum 300-s time resolution on all products will allow us the opportunity to phase-add data from separate Earth revolutions, should we wish to so, in addition to preserving the individual short-timescale information that may help us to deconvolve the effects of ice sheets, forests, and oceans.

J. Astron. Telesc. Instrum. Syst. 014003-24 Jan-Mar 2022 • Vol. 8(1) 


\section{Unparalleled Public Engagement Potential}

On Christmas Eve 1968, astronaut Bill Anders snapped a photograph of Earth rising above the lunar horizon from the Apollo spacecraft as it rounded the dark side of the Moon that has become one of the most iconic images of all time. Taken by the first of us to leave our planet behind and orbit another cosmic body, it ignited the human heart and mind in unexpected ways; young and old, scientists, philosophers, poets, environmentalists found new appreciation for the tiny, fragile, and beautiful world, devoid of cultural, political, or religious boundaries, enshrouded in a delicate atmosphere, the Spaceship Earth that we all call home. It reinforced that we share the only inhabited planet known, and it is our responsibility to nurture and protect it, for ourselves and future generations. In the years since, a variety of spacecraft have turned back to Earth, capturing images that continue to inspire and give perspective, from the stunning high-resolution Earth views from LRO, to the passage of the lunar shadow across Earth during the 2017 solar eclipse as seen from EPIC/DSCOVR, to Carl Sagan's famous "Pale Blue Dot" a blue-white "... lonely speck in the great enveloping cosmic dark" taken by Voyager-1, at $\sim 6$ billion km away.

Similarly, EarthShine images showing our planet through a series of illumination phases, including "new Earth," hold the promise of capturing the imagination of the general public, and could form the basis of a vibrant, inclusive communications, and public engagement program to engage the worldwide community. And it will do so by touching on multiple themes that each inspires tremendous public interest: human space exploration, stewardship of our home planet, Earth's place in the broader cosmos, and our future plans to search for life in the cosmos.

\section{Conclusion}

The confirmation of thousands of planets around a wide variety of stars, and the inference of billions more, powers NASA's enduring quest to search for and identify unambiguous signs of life elsewhere in the Universe. To accomplish this, we must launch enormous telescopes into space, equipped with cutting-edge direct imaging technology to block out light from the star, to collect spectra from the small fraction of light reflected from the planet. But since planets and their host stars are variable in space and time, it is not assured that we will recognize a habitable exoplanet when we see it. Further, the observatories that will make these observations will be complex and will have multiple operational considerations that are also complex. By considering Earth as an exoplanet covering key spectral regions and at a variety of illumination phases as seen from the lunar surface, both in "transit" and reflected light, and comparing with simultaneous data from NASA's Earth Science satellites, EarthShine can tease out features in the exoEarth spectral signature from environmental drivers unrelated to life. And this will provide us with a database that can be used to optimize the designs of any missions that seek to directly image other potentially Earth-like worlds. By observing the global Earth from a unique vantage point, EarthShine measurements will also contribute to important Earth Science goals by measuring Earth's radiation budget, accounting for energy exchanged between the Sun, planet, and space. NASA's return to the Moon offers the ideal opportunity to deploy a small, technologically mature instrument suite on the lunar surface to carry out these important science goals, while making the scientifically compelling and visually spectacular data quickly and broadly available to the astrophysics, Earth Science, and Planetary Science communities, as well as the Citizen Science community and the general public.

\section{Acknowledgments}

This research was supported by the NASA Goddard Internal Research and Development program. We would like to thank K. Jahoda, M. Eckart, W. Bruner, and R. Petre for useful discussions. K. Lindl and J. Yusko provided crucial editing and graphics support. PTB, APS and EW acknowledge support from NASA GSFC's Internal Research and Development program. SDG and GI are members of the Virtual Planetary Laboratory Team, a member of

J. Astron. Telesc. Instrum. Syst. 014003-25 Jan-Mar 2022 • Vol. 8(1) 
the NASA Nexus for Exoplanet System Science, funded via NASA Astrobiology Program Grant No. 80NSSC18K0829. P.P. was supported by NASA ISFM EIMM award, the NASA Cooperative Agreement 80GSFC21M0002, NASA Solar System Workings award No. 80NSSC21K0153, and NASA SSERVI award 80NSSC19M0217. TAL and TB also acknowledge support by NASA under award number 80GSFC21M0002. This work was partially supported by the GSFC Sellers Exoplanet Environments Collaboration (SEEC), which is funded through NASA ISFMs from the Planetary and Astrophysics Divisions.

\section{References}

1. B. King, "Sky \& telescope," 2018, https://skyandtelescope.org/astronomy-blogs/explorenight-bob-king/observing-earth-from-the-moon/.

2. C. Sagan et al., "A search for life on Earth from the Galileo spacecraft," Nature 365, 715-721 (1993).

3. P. R. Christensen and J. C. Pearl, "Initial data from the Mars Global Surveyor thermal emission spectrometer experiment: observations of the Earth," J. Geophys. Res. 102, 10875-10880 (1997).

4. T. A. Livengood et al., "Properties of an Earth-like planet orbiting a Sun-like star: Earth observed by the EPOXI mission," Astrobiology 11, 907-930 (2011).

5. N. B. Cowan et al., "Alien maps of an ocean-bearing world," Astrophys. J. 700, 915-923 (2009).

6. N. B. Cowan et al., "Rotational variability of Earth's polar regions: implications for detecting snowball planets," Astrophys. J. 731, 76 (2011).

7. Y. Fujii et al., "Colors of a second Earth. II. Effects of clouds on photometric characterization of Earth-like exoplanets," Astrophys. J. 738, 184 (2011).

8. T. D. Robinson et al., "Earth as an extrasolar planet: Earth model validation using EPOXI Earth observations," Astrobiology 11, 393-408 (2011).

9. T. D. Robinson et al., "Detection of ocean glint and ozone absorption using LCROSS Earth observations," Astrophys. J. 787, 171 (2014).

10. S. R. Kane et al., "Retrieval of planetary rotation and albedo from DSCOVR data," arXiv e-prints (2015).

11. A. Marshak et al., "Earth observations from DSCOVR EPIC instrument," Bull. Am. Meteorol. Soc. 99, 1829-1850 (2018).

12. W. Yang et al., "EPIC spectral observations of variability in Earth's global reflectance," Remote Sens. 10, 254 (2018).

13. S. Fan et al., "Earth as an exoplanet: a two-dimensional alien map," Astrophys. J. Lett. 882, L1 (2019).

14. M. C. Turnbull et al., "Spectrum of a habitable world: earthshine in the near-infrared," Astrophys. J. 644, 551 (2006).

15. T. Hearty et al., "Mid-infrared properties of disk averaged observations of Earth with AIRS," Astrophys. J. 693, 1763-1774 (2009).

16. D. C. Catling et al., "Exoplanet biosignatures: a framework for their assessment," Astrobiology 18, 709-738 (2018).

17. E. W. Schwieterman et al., "Exoplanet biosignatures: a review of remotely detectable signs of life," Astrobiology 18, 663-708 (2018).

18. A. Marshak, T. Varnai, and A. Kostinski. "Terrestrial glint seen from deep space: oriented ice crystals detected from the Lagrangian point," Geophys. Res. Lett. 44, 5197-5202 (2017).

19. L. Gu et al., "Earth as a proxy exoplanet: deconstructing and reconstructing spectrophotometric light curves," Astron. J. 161, 122 (2021).

20. M. B. Syal and P. H. Schultz, "Cometary impact effects at the Moon: implications for lunar swirl formation," Icarus 257, 194-206 (2015).

21. M. Benna et al., "Lunar soil hydration constrained by exospheric water liberated by meteoroid impacts," Nat. Geosci. 12, 333-338 (2019).

22. R. N. Clark, "Detection of adsorbed water and hydroxyl on the Moon," Science 326, 562 (2009). 
23. C. M. Pieters et al., "Character and spatial distribution of $\mathrm{OH} / \mathrm{H}_{2} \mathrm{O}$ on the surface of the Moon seen by M3 on Chandrayaan-1," Science 326, 568 (2009).

24. J. M. Sunshine et al., "Temporal and spatial variability of lunar hydration as observed by the deep impact spacecraft," Science 326, 565-568 (2009).

25. N. J. Woolf et al., "The spectrum of earthshine: a pale blue dot observed from the ground," Astrophys. J. 574, 430-433 (2002).

26. L. Arnold, "Earthshine observation of vegetation and implication for life detection on other planets. A review of 2001-2006 works," Space Sci. Rev. 135, 323-333 (2008).

27. E. Pallé et al., "Earth's transmission spectrum from lunar eclipse observations," Nature 459, 814-816 (2009).

28. J. H. Jiang et al., "Using deep space climate observatory measurements to study the Earth as an exoplanet," Astron. J. 156, 26 (2018).

29. S. Fan et al., "Earth as an exoplanet: a two-dimensional alien map," Astrophys. J. Lett. 882, L1 (2019).

30. H. Kawahara, "Global mapping of the surface composition on an exo-Earth using color variability," Astrophys. J. 894, 58 (2020).

31. M. Aizawa, H. Kawahara, and S. Fan, "Global mapping of an exo-Earth using sparse modeling," Astrophys. J. 896, 22 (2020).

32. H. Kawahara and K. Masuda, "Bayesian dynamic mapping of an exo-Earth from photometric variability," Astrophys. J. 900, 48 (2020).

33. S. A. Clough et al., "Atmospheric radiative transfer modeling: a summary of the AER codes," J. Quant. Spectrosc. Radiat. Transfer 91, 233 (2005).

34. D. P. Edwards, "GENLN2: a general line-by-line atmospheric transmittance and radiance model. Version 3.0 description and users guide," University Corporation for Atmospheric Research (1992).

35. T. D. Robinson and C. T. Reinhard, "Earth as an Exoplanet," in Planetary Astrobiology, V. S. Meadows et al., Eds., p. 379, University of Arizona Press (2020).

36. G. L. Villanueva et al., "Planetary spectrum generator: an accurate online radiative transfer suite for atmospheres, comets, small bodies and exoplanets," J. Quantum Spectrosc. Radiat. Transfer 217, 86-104 (2018).

37. G. L. Villanueva et al., "Fundamentals of the planetary spectrum generator," 2022 Edition, https://psg.gsfc.nasa.gov/help.php\#handbook.

38. K. F. Evans, "The spherical harmonics discrete ordinate method for three-dimensional atmospheric radiative transfer," J. Atmos. Sci. 55, 429-446 (1998).

39. S. L. Olson et al., "Atmospheric seasonality as an exoplanet biosignature," Astrophys. J. 858, L14 (2018).

40. G. Suissa et al., "The first habitable zone Earth-sized planet from TESS. III: climate states and characterization prospects for TOI-700d," Astron. J. 160, 118 (2020).

41. T. D. Robinson et al., "Titan solar occultation observations reveal transit spectra of a hazy world," Proc. Natl. Acad. Sci. U. S. A. 111, 9042 (2014).

42. E. J. R. Macdonald and N. B. Cowan, "An empirical infrared transit spectrum of Earth: opacity windows and biosignatures," Mon. Not. R. Astron. Soc. 489, 196 (2019).

43. G. Zotti et al., "The simulated sky: stellarium for cultural astronomy research," J. Skyscape Archaeol. 6(2), 221-258 (2021).

44. M. Horanyi et al., "A permanent, asymmetric dust cloud around the Moon," Nature 522, 324-326 (2015).

45. P. Pokorny et al., "Meteoroids at the Moon: orbital properties, surface vaporization, and impact ejecta production," J. Geophys. Res. Planets 124, 752 (2019).

46. M. Hollick and B. J. O'Brien, "Lunar weather measurements at three Apollo sits 19691976," Space Weather 11, 651 (2013).

47. J. R. Gaier and D. A. Jaworske, "Lunar dust on heat rejection system surfaces: problems and prospects," AIP Conf. Proc. 880, 27 (2007).

48. D. Koschny and E. Grun, "Impacts into ice-silicate mixtures: ejecta mass and size distributions," Icarus 154, 402 (2001).

49. E. Grun, M. Horanyi, and Z. Sternovsky, "The lunar dust environment," Planet. Space Sci. 59, 1672 (2011). 
50. O. E. Berg et al., "Preliminary results of a cosmic dust experiment on the Moon," Geophys. Research Lett. 1, 289 (1974).

51. T. J. Stubbs, R. R. Vondrak, and W. M. Farrell, "A dynamic fountain model for lunar dust," Adv. Space Res. 37, 59 (2006).

52. X. Wang et al., "Dust charging and transport on airless planetary bodies," Geophys. Research Lett. 43, 6103 (2016).

53. C. M. Katzan and J. L. Edwards, "Lunar dust transport and potential interactions with power system components," NASA Contractor Report 4404 (1991).

54. D. R. Criswell, "Horizon-glow and the motion of lunar dust," Astrophys. Space Sci. Libr. 37, 545 (1973).

55. A. Carroll et al., "Laboratory measurements of initial launch velocities of electrostatically lofted dust on airless planetary bodies," Icarus 352, 113972 (2020).

56. J. Marshall, D. Richard, and S. Davis, "Electrical stress and strain in lunar regolith stimulants," Planet. Space Sci. 59, 1744 (2011).

57. B. J. O'Brien and M. Hollick, "Sunrise-driven movements of dust on the Moon: Apollo 12 ground-truth measurements," Planet. Space Sci. 119, 194 (2015).

58. R. A. Howard et al., "Near-Sun observations of an F-corona decrease and K-corona fine structure," Nature 576, 232 (2019).

59. M. L. Kaiser, "The STEREO mission: an introduction," Space Sci. Rev. 136, 5 (2008).

60. J. M. Hahn et al., "Clementine observations of the zodiacal light and the dust content of the inner Solar System," Icarus 158, 360 (2002).

61. D. Nesvorny et al., "Cometary origin of the zodiacal cloud and carbonaceous micrometeorites. Implications for hot debris disks," Astrophys. J. 713, 816 (2010).

62. J. A. Burns, P. L. Larny, and S. Soter, "Radiation forces on small particles in the solar system," Icarus 40, 1-48 (1979).

63. E. Grun et al., "Collisional balance of the meteoritic complex," Icarus 62, 244 (1985).

64. D. Nesvorny et al., "Origin of the near-ecliptic circumsolar dust band," Astrophys. J. Lett. 679, L143 (2008).

65. D. Nesvorny et al., "Dynamical model for the zodiacal cloud and sporadic meteors," Astrophys. J. 743, 129 (2011).

66. D. Nesvorny et al., "Dynamics of dust particles released from Oort cloud comets and their contribution to radar meteors," Astrophys. J. 743, 37 (2011).

67. P. Pokorny et al., "Dynamical model for the toroidal sporadic meteors," Astrophys. J. 789, 25 (2014).

68. J. D. Carrillo-Sanchez et al., "Cosmic dust fluxes in the atmospheres of Earth, Mars, and Venus," Icarus 335, 113395 (2020).

69. E. L. Wilson et al., "Miniaturized laser heterodyne radiometer for measurements of $\mathrm{CO}_{2}$ in the atmospheric column," Appl. Phys. B 114, 385-393 (2014).

70. E. L. Wilson et al., "A $4 \mathrm{U}$ laser heterodyne radiometer for methane $\left(\mathrm{CH}_{4}\right)$ and carbon dioxide $\left(\mathrm{CO}_{2}\right)$ measurements from an occultation-viewing CubeSat," Meas. Sci. Technol. 28, 035902 (2017).

71. E. L. Wilson et al., "A portable miniaturized laser heterodyne radiometer (mini-LHR) for remote measurements of column $\mathrm{CH}_{4}$ and $\mathrm{CO}_{2}$," Appl. Phys. B 125, 211 (2019).

72. G. B. Clarke et al., "Uncertainty analysis for the miniaturized laser heterodyne radiometer (mini-LHR)," Meas. Sci. Technol. 25, 055204-055209 (2014).

73. H. R. Melroy et al., "Autonomous field measurements of $\mathrm{CO}_{2}$ in the atmospheric column with the miniaturized laser heterodyne radiometer (mini-LHR)," Appl. Phys. B 120, 609-615 (2015).

74. G. L. Villanueva et al., "Planetary spectrum generator (PSG)," 2016, https://ssed.gsfc.nasa .gov/psg/about.html.

75. G. L. Villanueva et al., "Strong water isotopic anomalies in the Martian atmosphere: probing current and ancient reservoirs," Science 348, 218-221 (2015).

76. M. Jhabvala et al., "Strained-layer-superlattice-based compact thermal imager for the International Space Station," Appl. Opt. 58, 5432 (2019).

77. A. R. Vasavada et al., "Lunar equatorial surface temperatures and regolith properties from the Diviner Lunar Radiometer Experiment," J. Geophys. Res. Planets 117, E00H18 (2012). 
Patricia T. Boyd is an astrophysicist at NASA's Goddard Space Flight Center. She has had leadership roles on a variety of space telescopes, including the Hubble, RXTE, Swift, Kepler, and TESS. She is studying planets around other stars, astrodynamics, accretion onto compact objects of stellar mass, and supermassive black holes in active galaxies. She is the chief of the Exoplanets and Stellar Astrophysics Laboratory at GSFC and the host of NASA's Curious Universe Science Podcast.

Emily L. Wilson specializes in the development of instruments that measure trace gases in Earth and planetary atmospheres. She has led the miniaturized laser heterodyne radiometer (miniLHR) development and deployments since 2009 (USA patent 8699029, 2014) including the MiniCarb CubeSat launched via SpaceX via the ISS in December 2019. She also serves as the associate deputy director for atmospheres in the Earth Science Division at NASA Goddard Space Flight Center.

Alan P. Smale has more than 30 years research experience in X-ray, optical, and IR astrophysics. $\mathrm{He}$ is studying variability in Galactic neutron star x-ray binaries, black hole candidates, and cataclysmic variables. He leads NASA's High Energy Astrophysics Science Archive Research Center and has substantial managerial, scientific, and technical expertise with mission ground systems, science operations, data processing pipelines, data archiving and analysis, and guest observer support (EXOSAT, BBXRT, ASCA, RXTE, Swift, Chandra, and many upcoming missions).

Pete Supsinskas is the associate program leader for the Lawrence Livermore National Laboratory's Space Science and Security Program and is responsible for the development of nano/small satellite technologies and spaceflight hardware for multiple government customers/ missions. Recent LLNL spaceflight missions he has led include the payloads for GEOStare 2 and the TacRL-2, both launched in 2021, and two additional payload programs in development, one of which will launch to the ISS in 2023.

Timothy A. Livengood is a planetary atmospheric scientist. He has been a coinvestigator with the Deep Impact EPOXI mission and with the Lunar Reconnaissance Orbiter's Lunar Exploration Neutron detector. He leads the development of the submillimeter solar observation lunar volatiles experiment, an instrument proposed to measure the concentration of water molecules in the tenuous lunar atmosphere.

Tilak Hewagama is a planetary scientist and instrument developer in the Detector Systems Branch at NASA's Goddard Space Flight Center. He served as a co-investigator with the EPOXI mission to study Earth as an exoplanet and transit photometry of exoplanet systems. $\mathrm{He}$ is the co-investigator on a range of instrument development programs and a lead developer of atmospheric radiative transfer algorithms in the analysis and interpretation of ground- and space-based spectroscopic observations.

Geronimo L. Villanueva is a planetary scientist at NASA's Goddard Space Flight Center who specializes in the search for water and organic molecules in planetary atmospheres. He is the principal investigator of the Planetary Spectrum Generator, the co-PI of the Comet Interceptor Mission, the co-investigator of the ExoMars/TGO mission, and a leader for Mars and Ocean Worlds studies for the James Webb Space Telescope.

Alexander Marshak is an atmospheric scientist at NASA's Goddard Space Flight Center. He conducts research on remote sensing of clouds, aerosols and blowing snow, cloud-aerosol interaction, and many aspects of atmospheric radiative transfer. He serves as the deputy project scientist for the Deep Space Climate Observatory satellite mission.

Nickolay A. Krotkov is an atmospheric scientist at NASA's Goddard Space Flight Center. He specializes in satellite retrievals and scientific understanding of Earth's trace gas species, such as sulfur dioxide, nitrogen dioxide, ozone, and aerosols. He is a deputy project scientist for NASA's Flagship Earth Observing System Atmospheric Chemistry Mission (Aura) and a member for Aura/OMI, SNPP/JPSS, DSCOVR/EPIC, EV-I/TEMPO, PACE science teams and NASA's Applied Science volcanic disasters response team. 
Petr Pokorny is an astrophysicist at NASA's Goddard Space Flight Center and The Catholic University of America, who specializes in numerical modeling of dust and meteoroids in the solar system, the effects of dust and meteoroid impacts on airless bodies and atmospheres of terrestrial planets, and the interpretation of meteor radar measurements.

Jay Bixler is a physicist at Lawrence Livermore National Laboratory working mainly on hyperspectral sensor development.

Jonathan D. Noland is currently a systems engineer at Lawrence Livermore National Laboratory. He has a background in aerospace engineering and space systems design.

Guru Ramu is a mechanical engineer at NASA's Goddard Space Flight Center, where he has led or supported the design, integration, and testing of a variety of instruments in development in the Earth Science and the Planetary Environments Laboratory.

Paul Cleveland is a thermal systems engineer at NASA's Goddard Space Flight Center, where he has led thermal modeling, integration, and launch support efforts on a variety of missions such as Hubble, JWST, TOPEX/Poseidon, and the Compton Gamma Ray Observatory.

John Ganino is a currently systems engineer at Lawrence Livermore National Laboratory with a background in aerospace program and project management.

Murzy Jhabvala is the chief engineer for detector development in the Instrument Systems and Technology Division at NASA's Goddard Space Flight Center. He leads the conceptualization and development of new and advanced infrared detectors, focal planes, and compact instruments. He has led device and instrument developments for the Cosmic Background Explorer, Solar and Heliospheric Observatory, Spitzer Space Telescope, Gravity Probe B, New Horizons, Landsats 8 and 9, James Webb Space Telescope, and the Compact Thermal Imager.

Elisa Quintana is an astrophysicist at NASA's Goddard Space Flight Center. She has held science and management roles on a variety of NASA missions, including Kepler, K2, TESS, and the Roman Space Telescope, and is the principal investigator of the Pandora Pioneer mission. She has worked on flight data calibration and analysis exoplanet discovery and validation, and studies the formation, dynamical stability, and habitability of extrasolar planetary systems.

Emily Gilbert is a PhD candidate at the University of Chicago. She studies magnetic activity of low-mass stars and searches for exoplanets using data from the Transiting Exoplanet Survey Satellite mission.

Knicole Colón is an astrophysicist at NASA's Goddard Space Flight Center. She has held various roles on several NASA missions, including Kepler, Hubble, TESS, JWST, and Pandora. She has additional experience working on ground-based telescope surveys to discover planets around other stars, and her research extends into studying the composition of exoplanet atmospheres.

Giada N. Arney is a research space scientist at the Planetary Systems Laboratory of NASA's Goddard Space Flight Center. Her research interests include astrobiology, planetary atmospheres, exoplanets, and future large space telescopes. She serves as a co-deputy principal investigator of the DAVINCI mission to Venus, whose goals include gathering data to help determine if Venus was more Earth-like in the past.

Shawn D. Domagal-Goldman is the branch head of the Planetary Systems at NASA's Goddard Space Flight Center, Greenbelt, MD, USA. He is an astrobiologist and a member of multiple interdisciplinary teams, including the Mars Curiosity rover team, and two future flagship telescope concepts: the Habitable Exoplanets Observatory and the Large UV-Optical-Infrared Surveyor. Both of these telescopes aim to search for potentially habitable exoplanets and to search for life on those worlds. His research focuses on the standards of evidence for biosignatures from such missions. 
Avi Mandell is an exoplanet scientist at the Planetary Systems Laboratory of NASA's Goddard Space Flight Center. His research focuses on the characterization of extrasolar planets and the formation and evolution of planetary systems, with the specific goal of understanding factors that determine whether a planetary system can form habitable planets and what the characteristics of these planets will be. He is the co-founder and previous director of the GSFC Sellers Exoplanet Environments Collaboration and the simulations subtask lead of the GSFC Exoplanet Spectroscopy Technologies team.

Tom Barclay is an associate research scientist at the University of Maryland, Baltimore County. He has served in various science and leadership roles on the Kepler and TESS space telescopes and has been involved in the development of new space mission concepts. His science interests focus on the detection and characterization exoplanets.

Marc Kuchner is an astrophysicist at NASA Goddard Space Flight Center who studies disks, exoplanets, and dynamics of solar system dust. He co-invented the band-limited coronagraph design used on the James Webb Space Telescope and received an early career achievement award from SPIE for this work. He founded two citizen science projects-Disk Detective and Backyard Worlds: Planet 9-and he serves as the citizen science officer for NASA's Science Mission Directorate.

Lesley Ott received her PhD from the University of Maryland and has since been working at NASA's Goddard Space Flight Center in the Global Modeling and Assimilation Office. She is a climate scientist focused on studying air pollution and greenhouse gases. Her work focuses on using a combination of satellite data and numerical models to better understand the sources and sinks of greenhouse gases and how the processes that control them may change over time. 